Elsevier required licence: (c) $<2017>$. This manuscript version is made available under the CC-BY-NC-ND 4.0 license http://creativecommons.org/licenses/bync-nd/4.0/ 


\title{
Performance Analysis of Fractional Frequency Reuse in Uplink Random Cellular Networks
}

\author{
Sinh Cong Lam* \\ University of Technology, Sydney \\ Center of Real Time Information Network \\ Faculty of Engineering and Information Technology \\ Kumbesan Sandrasegaran* \\ University of Technology, Sydney \\ Center of Real Time Information Network \\ Faculty of Engineering and Information Technology
}

\begin{abstract}
In this paper, we develop a network model based on 3GPP standards to analyse the performance of the uplink random cellular network using Frequency Reuse (FR) algorithms. The operation of FR is separated into two phases in which the Base Station (BS) measures the uplink SignalInterference-plus-Noise Ratio (SINR) to classify each user into either Cell-Center User (CCU) or Cell-Edge User (CEU) during the establishment phase. This is followed by the data transfer process between the user and it's serving BS during the communication phase. Compared with the related works, we propose the following novel approaches: (i) we define the two-phase operation for both CCU and CEU; (ii) the density of interfering users causing interference to the CEU under Strict FR is inversely proportional to a FR factor; (iii) the interference originating from CCUs and CEUs are evaluated separately. Although Strict FR provides more benefits for the user such as low power consumption and higher performance than Soft FR, the network using Soft FR can achieve a significantly higher cell data rate which is up to $58.96 \%$ higher than that using Strict FR. A very interesting phenomenon is found in this paper for a sparse Strict FR network with the density of BSs $\lambda=0.1 B S / \mathrm{km}^{2}$ in which the average uplink SINR of the user during establishment phase increases with the power control exponent while the corresponding average data rate of the CCU during the communication phase reduces. The paper also derives the approximation analytical approach using Gaussian Quadratures to obtain the close-form expressions of user's performance.le Keywords: Poisson Cellular Network, coverage probability, strict frequency reuse,soft frequency reuse, Rayleigh fading.
\end{abstract}

\footnotetext{
${ }^{*}$ Corresponding author

Email address: sinhcong.lam@student.uts.edu.au/lamsinhcong@gmail.com (Sinh Cong Lam)
} 


\section{Introduction}

In a Long Term Evolution (LTE) cellular network, InterCell Interference Coordination (ICIC) is a promising technique that can mitigate the InterCell Interference (ICI) and improve the spectral efficiency. Frequency Reuse (FR) 1 is an effective ICIC technique that utilises some restrictions 5 on power control and resource allocation to improve the performance of Cell-Edge Users (CEUs). Strict FR and Soft FR are the most common FR algorithms. Under these algorithms, the allocated resources are divided into $f_{c}+f_{e} \mathrm{RBs}$ in which each $\mathrm{RB}$ is defined as having a time duration of $0.5 \mathrm{~ms}$ and a bandwidth of $180 \mathrm{kHz}$ made up of 12 subcarriers with a subcarrier spacing of $15 \mathrm{kHZ}$. Under Strict FR, $f_{c}$ Cell-Center (CC) RBs are used as the common RBs and shared by all CellCenter Users (CCUs) of each cell while $f_{e}$ RBs are further partitioned into $\Delta$ Cell-Edge (CE) RB groups of $\frac{f_{e}}{\Delta}$ RBs where $\Delta$ is called a FR reuse factor. Therefore, each cell in the Strict FR network is allowed to use $f_{c}+\frac{f_{e}}{\Delta}$ RBs. Meanwhile, under Soft FR, each cell is allowed to reuse the whole RBs, i.e. $f_{e}+f_{c}$. Hence, Soft FR is expected to obtain higher spectral efficiency than Strict FR.

3GPP documents [2, 3] state that the operation of FR can be separated into two phases. During the first phase, called establishment phase, the BS measures the uplink SINR from the served user and compares it with the SINR threshold $T$ in order to classify each associated user into either CCU or CEU. After that, communication between the user and the BS is established and data is transferred during the second phase, called communication phase. While the data transmission between the user and the serving BS takes place continuously, the process of user classification depends on network operators and can be adjusted appropriately 4 .

In order to evaluate the performance of Strict FR, various types of network models such as hexagonal cell layout [1, Wyner model [5] and Point Poisson Process (PPP) model [6] have been utilized. While both hexagonal and Wyner model assume that the locations of BSs as well as the cell shapes are deterministic, the BSs in the PPP model are distributed randomly according to a 25 Spatial PPP model. Since the practical deployment of cellular networks depends on a number of practical considerations such as radio environment and user distribution, there is an increase in research works based on the PPP model to analyse the cellular network performance.

\subsection{Related works}

Although there is a lot of recent research work on the downlink performance of the FR algorithm so in the PPP network model [7, 8, 9], most of the important results on the PPP uplink network performance were presented for single user in [10, for multi-user in 11 and for a two-tier network in [12].

Although 10 presented a basic approach to analyse the performance of the uplink FR, the establishment and communication phases of the CCU were not distinguished which implied that 
the user transmits signals for user classification purpose and user data at the same time. In other words, the paper assumed that the user classification process takes place every time slot. Thus, the average coverage probability was defined as $\mathbb{P}(S I N R>\max (T, \hat{T}))$ in which $\hat{T}$ and $T$ are the coverage and SINR thresholds. However, in practical network, this assumption is not feasible because even the user can transmit the uplink control information and data during the same frame

40 [13, this assumption can result in a large increase in the volume of the control information in the network. Furthermore, the requirement for rapidly changing uplink transmit power at every time slot is challenging to both the BS and user.

Under Strict FR, when a user is defined as a CEU, it will be served on a CE RB, which is a private resource within a group of $\Delta$ cells. Therefore, the density of interfering users in the case of ${ }_{45} \mathrm{CEU}$ is $\lambda / \Delta$ [14], in which $\lambda$ is the density of BSs. However, this point was not discussed in [10].

Under Soft FR, each user experiences interference from a set of CCUs $\theta_{c}$ and a set of CEUs $\theta_{e}$. By introducing a constant coefficient, $\theta_{c}$ and $\theta_{e}$ were consolidated [7, 10, 15, to calculate the total interference in the network. However, in the PPP network model, since each BS is distributed randomly and completely independent of other BSs, each BS in $\theta_{c}$ is independently

so located compared to other BSs in $\theta_{e}$. Therefore, $\theta_{c}$ and $\theta_{e}$ should not be consolidated [16].

In recent our work [17, the downlink random cellular network using FR was modelled and analysed under Rayleigh-Lognormal fading environment. This paper was based on the approaches in previous works such as 7,15 , thus there were some limitations such as: (i) the two-phase operation of FR was only defined for the CEU; (ii) the constant coefficient approach was used

${ }_{55}$ to represent the total network interference, which was not correct for the PPP network layout as discussed in the previous paragraph. Furthermore, the operations of FR in downlink and uplink also have differences such as: (i) although in both downlink and uplink, the CEU during the communication phase is served on the different RB with the establishment phase, it's interfering sources between two phases are the same in the case of downlink and completely different in the

${ }_{60}$ case of uplink; (ii) the uplink uses the power control exponent to adjust the user's transmission power. Hence, the analytical and approximation approach in this paper and in [17] have significant differences.

In [11] and 12, only the communication phase of the FR algorithm was considered. Thus, the average coverage probability is defined as $\mathbb{P}(S I N R>\hat{T})$. To the best of our knowledge, recent research on this topic has been based on the hexagonal model [18, 19, 20. Hence, there should be more research on the uplink PPP network using FR.

\subsection{Contributions}

In this paper, we develop a uplink PPP model based on 3GPP recommendations for the cellular network using Strict FR and Soft FR. The main differences in the network model between this 
work and the related works are summarized as below:

- For CCU under both Strict FR and Soft FR: This paper follows the 3GPP recommendations to separate the establishment phase and communication phase. Thus, the average coverage probability of the CCU is defined as the conditional probability of SINR during the communication phase under the condition on SINR during the establishment phase.

- For CEU under Strict FR: This paper bases on the fact that the CE RB is the private resource within a group of $\Delta$ cells [1, and consequently the interfering users is distributed with a density of $\lambda / \Delta$ in which $\lambda$ is the density of BSs.

- For Soft FR: This paper develops our work for the downlink cellular network using Soft FR [16], which separately evaluated interference from the sources transmitting on the CC and CE powers.

We derive the highly tractable expressions of network performance in terms of the probabilities, in which the user is served as a CCU and CEU, average transmit power and coverage probability of the user, as well as average network data rate. We utilise the Gaussian Quadratures to approximate the complex expressions of the network performance by the simple finite sums, which can be considered as the closed-form expressions.

The performance of Strict FR and Soft FR are analysed and compared together through the paper. While the Strict FR outperforms Soft FR in terms of user performance, Soft FR can achieve higher cell data rate than Strict FR. Furthermore, we study three scenarios of the cellular networks with different densities of BSs which correspond to sparse $\left(\lambda=0.1 \mathrm{BS} / \mathrm{km}^{2}\right)$, medium 90 dense $\left(\lambda=0.5 \mathrm{BS} / \mathrm{km}^{2}\right)$ and dense $\left(\lambda=1 \mathrm{BS} / \mathrm{km}^{2}\right)$ networks. While the results in [10, 21] concluded that the average transmit power and coverage probability continuously reduce when the power control exponent increases, our findings state that the upward and downward trends of these parameters depend on both the density of BSs and the power control exponent. Furthermore, a very interesting phenomenon is found in this paper for a sparse network using Strict FR with

95 the density of BSs $\lambda=0.1 B S / \mathrm{km}^{2}$, in which the average uplink SINR of the user during the establishment phase increases with the power control exponent while the corresponding average data rate of the CCU during the communication phase reduces. This finding has not been discussed in previous works since in those works, the establishment phase and communication phase were not separated. 


\section{System model}

\subsection{Network topology}

We consider the uplink model of a PPP cellular network, in which both BSs and users are distributed according to a spatial PPP model with densities of $\lambda\left(B S s / \mathrm{km}^{2}\right)$ and $\lambda^{(u)}\left(u s e r s / \mathrm{km}^{2}\right)$ respectively. We assume that $\lambda^{(u)} \gg \lambda$, so that all the BSs and RBs are activated to serve the associated users. We also assume that on a given time slot, each RB is only allocated to a user per cell. Based on these assumptions, the density of users that transmit on the same RB at the same time slot is exactly the same as the density of BSs.

The typical user is allowed to associate with the nearest BS. We denote $r$ as the distance from the user to the serving BS, which is a random variable whose Probability Density Function (PDF) is given by [10]:

$$
f_{R}(r)=2 \pi \lambda r e^{-\lambda \pi r^{2}}
$$

In the uplink network, each mobile user's transmit power is controlled to achieve a desired received signal power $P$ at the serving BS. Conventionally, the user transmit power is adjusted based on pathloss-inversion of a form $\operatorname{Pr}^{\alpha \epsilon}$ [10, 21], in which $\alpha$ and $\epsilon$ are pathloss and power control exponents, $(\alpha \in[0,1])$. In a particular network, the selection of $\epsilon$ is based upon interference, channel fading and battery consumption. The average transmit power of the CCU is given by

$$
E\left[P_{r}\right]=\int_{0}^{\infty} 2 \pi \lambda r^{\epsilon \alpha+1} e^{-\pi \lambda r^{2}} d r
$$

\subsection{Frequency Reuse Algorithm}

In this paper, we investigate on a two-phase operation of FR algorithm according to 3GPP documents 13, 22. These documents also states that the user can transmit both uplink control information and data during the same frame. Hence, there is no difference when the BS measures SINR from the control channel and data channel. The two-phase operation of FR algorithm is described as below:

- During the establishment phase, the BS uses the SINR on the uplink CC RB to classify the corresponding user into either CCU or CEU. In this phase, the average probabilities in which the user is classified as a CCU and CEU, (CCU and CEU classification probabilities for simplicity), are analysed.

- During the communication phase, the user acts as either CCU or CEU. In this phase, the performance of the user and network are evaluated with respect to the power control exponent, SINR threshold and density of BSs. Furthermore, the average transmit power of the user is also discussed. 
Since a CE power of the CEU is greater than a CC power of the CCU, the transmit power of user $z$ is denoted by $P^{(z)}=\phi^{(z)} \operatorname{Pr}^{\alpha \epsilon}$ where $z=(c, e)$ correspond to the CCU and CEU, $\phi^{(e)}=\phi$ $(\phi>1)$ is a transmit ratio between the $\mathrm{CE}$ and $\mathrm{CC}$ powers, and $\phi^{(c)}=1$. Each RB can be used at adjacent cells at the same time, and consequently each user can experience interference from other users who are at adjacent cells and transmitting on the same RB. The set of users that may create interference to the uplink of the user is denoted by $\theta_{F R}^{(z)}$. With assumption that each RB is allocated to a user, each user in $\theta_{S t r}^{(e)}$ is located independently to others in $\theta_{S t r}^{(c)}$. We denote $I_{F R}^{(z)}$ as the corresponding interference, in which $F R=(S t r, S o f)$ correspond to Strict FR and Soft FR.

The CCU during the communication phase is served on the same RB, and consequently experiences interference originating the same users with the establishment phase. Meanwhile, since the $\mathrm{CEU}$ during the communication phase is served on different RB compared to the establishment phase and combination with the assumption that each RB is only allocated to a user during a given timeslot, the interference sources between two phases are completely different.

- Under Strict FR: Since the CCUs do not share their own RBs with the CEUs and vice versa, the $I_{S t r}^{(z)}$ originates from either CCUs or CEUs. The power of interference $I_{S t r}^{(z)}$ at the serving BS of user $z$ is

$$
I_{S t r}^{(z)}=\sum_{j \in \theta_{S t r}^{(z)}} P_{j}^{(z)} g_{j z} d_{j z}^{-\alpha}
$$

in which $g_{j z}$ and $d_{j z}$ are the power gain and distance from interfering user $j$ to the serving BS of user $z$.

During the establishment phase, since the BS measures the uplink SINR on the CC RBs which are common RBs and shared by all BSs, the density of interfering users of the measured SINR during the establishment phase and CCU during communication phase is $\lambda$. In the case of CEU, since the CEU is served on a $\mathrm{CE} \mathrm{RB,} \mathrm{which} \mathrm{is} \mathrm{a} \mathrm{private} \mathrm{RB} \mathrm{within} \mathrm{a} \mathrm{group} \mathrm{of} \Delta$ cells, the density of interfering users in this case is only $\frac{\lambda}{\Delta}$.

- Under Soft FR: Since each cell can reuse all RBs, each CC RB as well as CE RB may experience interference from both CCUs and CEUs whose densities are $\frac{\Delta-1}{\Delta} \lambda$ and $\frac{1}{\Delta} \lambda[16$. In this case, $I_{\text {Sof }}^{(z)}$ is given by

$$
I_{\text {Sof }}^{(z)}=\sum_{j \in \theta_{\text {Sof }}^{(c)}} P_{j}^{(c)} g_{j z} d_{j z}^{-\alpha}+\sum_{j \in \theta_{\text {Sof }}^{(e)}} P_{j}^{(e)} g_{j z} d_{j z}^{-\alpha}
$$

Eq. 4 represents interference of the measured SINR during the establishment phase, and both CCU and CEU during the communication phase. 
The received SINR at the BS from user $z$ is given by

$$
\operatorname{SINR}\left(\phi^{(z)}, \epsilon\right)=\frac{\phi^{(z)} \operatorname{Pgr}^{\alpha(\epsilon-1)}}{\sigma^{2}+I_{F R}^{(z)}\left(\phi^{(z)}\right)}
$$

in which $\sigma^{2}$ is the Gaussian noise power, $g$ and $r$ is the power gain and distance from user $z$ to the serving BS. In this paper, the channel fading has a Rayleigh distribution with an average power of 1. Hence, $g$ has an exponential distribution and $E\left[g_{j z}\right]=1$.

\subsubsection{User Classification Probability}

The user is defined as a CCU if it's uplink SINR during establishment phase, denoted by $\operatorname{SIN} R^{(o)}(1, \epsilon)$, is greater than the SINR threshold $T$. The average probabilities, in which the

150 typical user is served as a CCU under Strict FR and Soft FR ( CCU classification probabilities), are given by Remark 2.1 and Remark 2.2 .

Remark 2.1. (Strict FR, CCU Classification Probability): The probability $A_{S t r}^{(c)}(T, \epsilon \mid r)$ that a user at a distance $r$ from it's serving $B S$ is defined as a $C C U$ is obtained by evaluating the conditional probability $\mathbb{P}\left(\operatorname{SIN} R^{(o)}(1, \epsilon)>T\right)$.

$$
A_{S t r}^{(c)}(T, \epsilon \mid r)=e^{-\frac{T}{S N R} r^{\alpha(1-\epsilon)}} \mathscr{L}_{I_{\theta}^{(o c)}}\left(s_{1}, \lambda\right)
$$

and thus CCU classification probability is given by

$$
\begin{aligned}
A_{S t r}^{(c)}(T, \epsilon) & =\int_{0}^{\infty} A_{S t r}^{(c)}(T, \epsilon \mid r) f_{R}(r) d r \\
& =\int_{0}^{\infty} v(T) \mathscr{L}_{I_{\theta}^{(o c)}}\left(s_{1}, \lambda\right) d r
\end{aligned}
$$

in which $f_{R}(r)$ is defined in Equation (1), $v(T)=2 \pi \lambda r e^{-\pi \lambda r^{2}-\frac{T}{S N R} r^{\alpha(1-\epsilon)}}, S N R=\frac{P}{\sigma^{2}}, s_{1}=\operatorname{Tr}^{-\alpha \epsilon}$ and $\mathscr{L}_{I_{\theta}^{(o c)}}\left(s_{1}, \lambda\right)=e^{-2 \pi \lambda r^{2} \int_{1}^{\infty}\left(1-\int_{0}^{\infty} \frac{\pi \lambda t e^{-\lambda \pi t^{2}}}{1+s_{1} t^{\alpha \epsilon} x^{-\alpha}} d t\right) x d x}$.

This remark can be proved based on Theorem 1 in [10] or using the results of Appendix A with $155 \phi=1$.

Remark 2.2. (Soft FR, CCU Classification Probability): The probability $A_{\text {Sof }}^{(c)}(T, \epsilon \mid r)$ that a user at a distance $r$ from it's serving $B S$ is defined as a $C C U$ is given by

$$
A_{S o f}^{(c)}(T, \epsilon \mid r)=e^{-\frac{T r^{\alpha(1-\epsilon)}}{S N R}} \mathscr{L}_{I_{\theta}^{(o c)}}\left(s_{1}, \frac{\Delta-1}{\Delta} \lambda\right) \mathscr{L}_{I_{\theta}^{(o c)}}\left(\phi s_{1}, \frac{1}{\Delta} \lambda\right)
$$

and thus $C C U$ classification probability is given by

$$
A_{\text {Sof }}^{(c)}(T, \epsilon)=\int_{0}^{\infty} v(T) \mathscr{L}_{I_{\theta}^{(o c)}}\left(s_{1}, \frac{\Delta-1}{\Delta} \lambda\right) \mathscr{L}_{I_{\theta}^{(o c)}}\left(\phi s_{1}, \frac{1}{\Delta} \lambda\right) d r
$$

This remark is proved by Appendix A.

Proposition 2.3. The CCU classification probabilities in Remark 2.1 and 2.2 can be approximated 
by using the Gauss Quadratures.

$$
\begin{aligned}
& A_{\text {Str }}^{(c)}(T, \epsilon) \approx \sum_{j=0}^{N_{G L}} e^{-\frac{T}{S N R} \zeta_{j}^{\alpha(1-\epsilon)}} \mathscr{L}_{I_{\theta}^{(o c)}}^{(j)}\left(T \zeta_{j}^{-\alpha \epsilon}, \lambda\right) \\
& A_{\text {Sof }}^{(c)}(T, \epsilon) \approx \sum_{j=0}^{N_{G L}} e^{-\frac{T}{S N R} \zeta_{j}^{\alpha(1-\epsilon)}} \mathscr{L}_{I_{\theta}^{(o c)}}^{(j)}\left(T \zeta_{j}^{-\alpha \epsilon}, \frac{\Delta-1}{\Delta} \lambda\right) \mathscr{L}_{I_{\theta}^{(o c)}}^{(j)}\left(\phi T \zeta_{j}^{-\alpha \epsilon}, \frac{1}{\Delta} \lambda\right)
\end{aligned}
$$

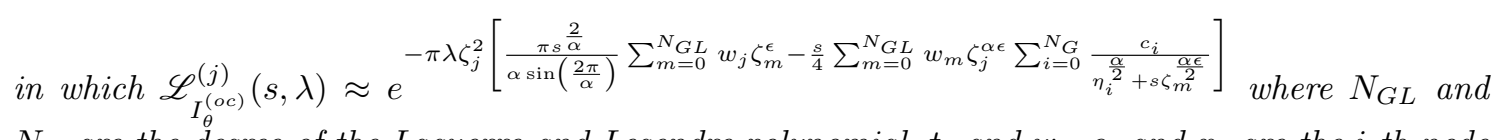
$N_{G}$ are the degree of the Laguerre and Legendre polynomial, $t_{i}$ and $w_{i}, c_{i}$ and $x_{i}$ are the $i$-th node and weight, abscissas and weight of the corresponding quadratures; $\zeta_{j}=\sqrt{\frac{t_{j}}{\pi \lambda}}$.

${ }_{160}$ For both Gauss-Laguerre and Gauss-Legendre Quadrature, higher degrees of the polynomials give better approximation. The values of $t_{i}$ and $w_{1}, c_{i}$ and $t_{i}$ can be found from [23].

Proof: See Appendix B

It is clear that the CEU classification probability can be obtained by $A_{F R}^{(e)}(T, \epsilon)=1-A_{F R}^{(c)}(T, \epsilon)$.

\section{Average Coverage Probability}

\subsection{Average Coverage Probability Definition}

In this section, we derive the performance expression of the CCU and CEU in the FR network, which follows $3 \mathrm{GPP}$ recommendations. In case of the $\mathrm{CCU}$, the $\mathrm{CCU}$ is covered by the network when it's uplink SINR at the serving BS is greater than the SINR threshold $T$ during the establishment phase and the coverage threshold $\hat{T}$ during the communication phase. Hence, the average coverage probability is defined as:

$$
\mathcal{P}^{(c)}(T, \epsilon)=\mathbb{P}\left(\operatorname{SINR}(1, \epsilon)>\hat{T} \mid \operatorname{SIN} R^{(o)}(1, \epsilon)>T\right)
$$

Similarity, in case of the CEU, the average coverage probability is defined by the following equation:

$$
\mathcal{P}^{(e)}(T, \epsilon)=\mathbb{P}\left(\operatorname{SINR}(\phi, \epsilon)>\hat{T} \mid S I N R^{(o)}(1, \epsilon)<T\right)
$$

The definition of the average CCU coverage probability differs from the previous works such as in [10] since those works did not separate the establishment and communication phase.

\subsection{Average Coverage Probability of $C C U$ and $C E U$}

Theorem 3.1. (Strict FR, CCU) The average coverage probability of the $C C U$ is given by

$$
\mathcal{P}_{S t r}^{(c)}(T, \epsilon)=\frac{\int_{0}^{\infty} v(T+\hat{T}) \mathscr{L}\left(s_{1}, s_{2}, \lambda\right) d r}{\int_{0}^{\infty} v(T) \mathscr{L}_{I_{\theta}^{(o c)}}\left(s_{1}\right) d r}
$$

where $\mathscr{L}_{I_{\theta}^{(o c)}}\left(s_{1}\right)$ and $v(T)$ are defined in Equation $(7) ; s_{1}=T r^{-\alpha \epsilon} ; s_{2}=\hat{T} r^{-\alpha \epsilon}$ and

$\mathscr{L}\left(s_{1}, s_{2}, \lambda\right)=e^{-2 \pi \lambda r^{2} \int_{1}^{\infty}\left[1-\int_{0}^{\infty} \frac{\pi \lambda t e^{-\pi \lambda t^{2}}}{\left(1+s_{1} t^{\alpha \epsilon} x^{-\alpha}\right)\left(1+s_{2} t^{\alpha \epsilon} x^{-\alpha}\right)} d t\right] x d x}$. 
Proof: See Appendix C

Proposition 3.2. The average coverage probability in Equation (14) can be approximated by

$$
\mathcal{P}_{S t r}^{(c)}(T, \epsilon) \approx \frac{\sum_{j=1}^{N_{G L}} w_{j} e^{-\frac{(T+\hat{T})}{S N R} \zeta_{j}^{\alpha(1-\epsilon)}} \mathscr{L}^{(j)}\left(\hat{T} \zeta_{j}^{-\alpha \epsilon}, T \zeta_{j}^{-\alpha \epsilon}, \lambda\right)}{\sum_{j=1}^{N_{G L}} w_{j} e^{-\frac{T}{S N R} \zeta_{j}^{(1-\epsilon)}} \mathscr{L}_{I_{\theta}^{(o c)}}^{(j)}\left(T \zeta_{j}^{-\alpha \epsilon}\right)}
$$

in which $\mathscr{L}^{(j)}\left(s_{1}, s_{2}, \lambda\right) \approx e^{-\pi \lambda \zeta_{j}^{2} \sum_{m=1}^{N_{G L}} w_{m}\left(I_{0}\left(\zeta_{m}\right)-I_{1}\left(\zeta_{m}\right)\right)}, I_{0}(t)=\frac{2 t^{\epsilon}}{\alpha} \frac{s_{1}^{1+\frac{2}{\alpha}}-s_{2}^{1+\frac{2}{\alpha}}}{s_{1}-s_{2}} \frac{\pi}{\sin \left(\frac{2 \pi}{\alpha}\right)}$ and $I_{1}(t)=$ $\sum_{i=1}^{N_{G}} \frac{c i}{2} \frac{\left(s_{1}+s_{2}\right) t^{\alpha \epsilon} \eta_{i}^{\frac{\alpha}{2}}+s_{1} s_{2} t^{\alpha \epsilon}}{\left(\eta_{i}^{\frac{\alpha}{2}}+s_{1} t^{\alpha \epsilon}\right)\left(\eta_{i}^{\frac{\alpha}{2}}+s_{2} t^{\alpha \epsilon}\right)} ; \eta_{i}=\frac{x_{i}+1}{2}$.

Proof: See Appendix D

Theorem 3.3. (Strict FR, CEU) The average coverage probability of the CEU is given by

$$
\mathcal{P}_{S t r}^{(e)}(T, \epsilon)=\frac{\int_{0}^{\infty} v\left(\frac{\hat{T}}{\phi}\right) \mathscr{L}_{I_{\theta}^{(o c)}}\left(s_{2}, \frac{\lambda}{\Delta}\right)\left(1-e^{-\frac{T}{S N R} r^{\alpha(1-\epsilon)}} \mathscr{L}_{I_{\theta}^{(o c)}}\left(s_{1}, \lambda\right)\right) d r}{1-\int_{0}^{\infty} v(T) \mathscr{L}_{I_{\theta}^{(o c)}}\left(s_{1}\right) d r}
$$

Proof: See Appendix E

Using the same approach in Appendix B and Appendix D, we obtain

$$
\mathcal{P}_{S t r}^{(e)}(T, \epsilon) \approx \frac{\sum_{j=1}^{N_{G L}} w_{j} e^{-\frac{\hat{T}}{\phi S N R} \zeta_{j}^{\alpha(1-\epsilon)}} \mathscr{L}_{I_{\theta}^{(o c)}}^{(j)}\left(\hat{T} \zeta_{j}^{-\alpha \epsilon}, \frac{\lambda}{\Delta}\right)\left(1-e^{-\frac{T}{S N R} \zeta_{j}^{\alpha(1-\epsilon)}} \mathscr{L}_{I_{\theta}^{(o c)}}^{(j)}\left(T \zeta_{j}^{-\alpha \epsilon}, \lambda\right)\right)}{1-\sum_{j=1}^{N_{G L}} w_{j} e^{-\frac{T}{S N R} \zeta_{j}^{\alpha(1-\epsilon)}} \mathscr{L}_{I_{\theta}^{(j)}}^{(j)}\left(T \zeta_{j}^{-\alpha \epsilon}, \lambda\right)}
$$

Theorem 3.4. (Soft FR, CCU) The average coverage probability of the $C C U$ is given by

$$
\mathcal{P}_{\text {Sof }}^{(c)}(T, \epsilon)=\frac{\int_{0}^{\infty} v(T+\hat{T}) \mathscr{L}\left(s_{1}, s_{2}, \frac{\Delta-1}{\Delta} \lambda\right) \mathscr{L}\left(\phi s_{1}, \phi s_{2}, \frac{\lambda}{\Delta}\right) d r}{\int_{0}^{\infty} v(T) \mathscr{L}_{I_{\theta}^{(o c)}}\left(s_{1}, \frac{\Delta-1}{\Delta} \lambda\right) \mathscr{L}_{I_{\theta}^{(o c)}}\left(\phi s_{1}, \frac{1}{\Delta} \lambda\right) d r}
$$

The approximated value of $\mathcal{P}_{\text {Sof }}^{(c)}(T, \epsilon)$ is given by

$$
\frac{\sum_{j=1}^{N_{G L}} w_{j} e^{-\frac{T+\hat{T}}{S N R} \zeta_{j}^{\alpha(1-\epsilon)}} \mathscr{L}^{(j)}\left(T \zeta_{j}^{-\alpha \epsilon}, \hat{T} \zeta_{j}^{-\alpha \epsilon}, \frac{\Delta-1}{\Delta} \lambda\right) \mathscr{L}^{(j)}\left(\phi T \zeta_{j}^{-\alpha \epsilon}, \phi \hat{T} \zeta_{j}^{-\alpha \epsilon}, \frac{\lambda}{\Delta}\right)}{\sum_{j=1}^{N_{G L}} w_{j} e^{-\frac{T}{S N R} \zeta_{j}^{(1-\epsilon)}} \mathscr{L}_{I_{\theta}^{(o)}}^{(j)}\left(T \zeta_{j}^{-\alpha \epsilon}, \frac{\Delta-1}{\Delta} \lambda\right) \mathscr{L}_{I_{\theta}^{(o o)}}^{(j)}\left(\phi T \zeta_{j}^{-\alpha \epsilon}, \frac{1}{\Delta} \lambda\right)}
$$

Proof: See Appendix F

Theorem 3.5. (Soft FR, CEU) The average coverage probability of the CEU is given by

$$
\mathcal{P}_{\text {Sof }}^{(e)}(T, \epsilon)=\frac{\left[\begin{array}{c}
\int_{0}^{\infty} v\left(\frac{\hat{T}}{\phi}\right) \mathscr{L}_{I_{\theta}^{(o c)}}\left(\frac{s_{2}}{\phi}, \frac{\Delta-1}{\Delta} \lambda\right) \mathscr{L}_{I_{\theta}^{(o c)}}\left(s_{2}, \frac{1}{\Delta} \lambda\right) \\
\times\left(1-e^{-\frac{T r^{\alpha(1-\epsilon)}}{S N R}} \mathscr{L}_{I_{\theta}^{(o c)}}\left(s_{1}, \frac{\Delta-1}{\Delta} \lambda\right) \mathscr{L}_{I_{\theta}^{(o c)}}\left(\phi s_{1}, \frac{1}{\Delta} \lambda\right)\right)
\end{array}\right]}{1-\int_{0}^{\infty} v(T) \mathscr{L}_{I_{\theta}^{(o c)}}\left(s_{1}, \frac{\Delta-1}{\Delta} \lambda\right) \mathscr{L}_{I_{\theta}^{(o c)}}\left(\phi s_{1}, \frac{1}{\Delta} \lambda\right) d r}
$$

and it's approximation is derived by

$$
\mathcal{P}_{\text {Sof }}^{(e)}(T, \epsilon) \approx \frac{\sum_{j=1}^{N_{G L}} w_{j}\left[\begin{array}{c}
e^{-\frac{\hat{T}}{\phi S N R} \zeta_{j}^{\alpha(1-\epsilon)}} \mathscr{L}_{I_{\theta}^{(o c)}}^{(j)}\left(\frac{\hat{T} \zeta_{j}^{-\alpha \epsilon}}{\phi}, \frac{\Delta-1}{\Delta} \lambda\right) \mathscr{L}_{I_{\theta}^{(o c)}}^{(j)}\left(\hat{T} \zeta_{j}^{-\alpha \epsilon}, \frac{1}{\Delta} \lambda\right) \\
\times\left(1-e^{-\frac{T}{S N R} \zeta_{j}^{\alpha(1-\epsilon)}} \mathscr{L}_{I_{\theta}^{(o c)}}^{(j)}\left(T \zeta_{j}^{-\alpha \epsilon}, \frac{\Delta-1}{\Delta} \lambda\right) \mathscr{L}_{I_{\theta}^{(o c)}}^{(j)}\left(\phi T \zeta_{j}^{-\alpha \epsilon}, \frac{1}{\Delta} \lambda\right)\right)
\end{array}\right]}{1-\sum_{j=1}^{N_{G L}} w_{j} e^{-\frac{T}{S N R} \zeta_{j}^{\alpha(1-\epsilon)}} \mathscr{L}_{I_{\theta}^{(j)}}^{(j)}\left(T \zeta_{j}^{-\alpha \epsilon}, \frac{\Delta-1}{\Delta} \lambda\right) \mathscr{L}_{I_{\theta}^{(j o)}}^{(j)}\left(\phi T \zeta_{j}^{-\alpha \epsilon}, \frac{1}{\Delta} \lambda\right)}
$$

Proof: See Appendix G 


\subsection{Average Coverage Probability of a Typical User}

In the cellular network, a typical user can be classified as a CCU with the transmit power $\operatorname{Pr}^{\alpha \epsilon}$ or CEU with the transmit power $\phi P r^{\alpha \epsilon}$. Therefore, evaluating the performance of the typical user can bring an overall view on trends of the network performance as well as user's power consumption. The transmit power of the typical user at a distance from it's serving BS is obtained by

$$
\begin{aligned}
P_{u}(r) & =\mathbb{P}\left(\operatorname{SINR}^{(o)}(1, \epsilon)>T \mid r\right) P^{(c)}+\mathbb{P}\left(\operatorname{SINR}^{(o)}(1, \epsilon)<T \mid r\right) P^{(e)} \\
& =\operatorname{Pr}^{\alpha \epsilon} A_{F R}^{(c)}(T, \epsilon \mid r)+\phi \operatorname{Pr}^{\alpha \epsilon}\left(1-A_{F R}^{(c)}(T, \epsilon \mid r)\right)
\end{aligned}
$$

in which $\mathbb{P}\left(S I N R^{(o)}(1, \epsilon)>T \mid r\right)$ and $\mathbb{P}\left(S I N R^{(o)}(1, \epsilon)<T \mid r\right)$ are the probabilities where the user at a distance $r$ from it's serving BS is classified as a CCU and CEU, $P^{(c)}$ and $P^{(e)}$ are corresponding transmit powers; $A_{F R}^{(c)}(T, \epsilon \mid r)$ defined in Remark 2.1 and 2.2

Thus, the average transmit power of the typical user is obtained by

$$
\overline{P_{u}}=\int_{0}^{\infty} 2 \pi \lambda r e^{-\pi \lambda r^{2}} \operatorname{Pr}^{\alpha \epsilon} A_{F R}^{(c)}(T, \epsilon \mid r)+\phi P r^{\alpha \epsilon} A_{F R}^{(e)}(T, \epsilon \mid r) d r
$$

Employing a change of variable $t=\pi \lambda r^{2}$ and using Gauss-Laguerre Quadrature, the average transmit power is approximated by

$$
\overline{P_{u}} \approx \sum_{j=1}^{N_{G L}} \omega_{j} P \zeta_{j}^{\frac{\alpha \epsilon}{2}} A_{F R}^{(c)}\left(T, \epsilon \mid r=\zeta_{j}\right)+\phi P \zeta_{j}^{\alpha \epsilon} A_{F R}^{(e)}\left(T, \epsilon \mid r=\zeta_{j}\right)
$$

where $\zeta_{j}=\sqrt{\frac{t_{j}}{\pi \lambda}} ; N_{G L}$ is the degree of the Laguerre polynomial, $t_{i}$ and $w_{i}$ are the $i$-th node and weight of the corresponding quadrature.

The average coverage probability of the typical user is given by:

$$
\begin{aligned}
\mathcal{P}_{F R}(T, \epsilon \mid r)= & \mathbb{P}\left(\operatorname{SIN} R^{(o)}(1, \epsilon)>T \mid r\right) \mathbb{P}(\operatorname{SINR}(1, \epsilon)>T \mid r) \\
& +\mathbb{P}\left(\operatorname{SINR} R^{(o)}(1, \epsilon)<T \mid r\right) \mathbb{P}(\operatorname{SINR}(\phi, \epsilon)<T \mid r)
\end{aligned}
$$

in which $\mathbb{P}(\operatorname{SINR}(1, \epsilon)>T \mid r)$ and $\mathbb{P}(\operatorname{SINR}(\phi, \epsilon)<T \mid r)$ are the coverage probabilities of the CCU 185 and CEU whose distances to the serving BSs are $r$.

Therefore, the average coverage probability of the typical users is

$$
\begin{aligned}
\mathcal{P}_{F R}(T, \epsilon) & =\int_{0}^{\infty} 2 \pi \lambda r e^{-\pi \lambda r^{2}}\left[A_{F R}^{(c)}(T, \epsilon \mid r) \mathcal{P}_{F R}^{(c)}(T, \epsilon \mid r)+\left(1-A_{F R}^{(c)}(T, \epsilon \mid r)\right) \mathcal{P}_{F R}^{(e)}(T, \epsilon \mid r)\right] d r \quad(25) \\
& \approx \sum_{j=1}^{N_{G L}} \omega_{i}\left[A_{F R}^{(c)}\left(T, \epsilon \mid r=\zeta_{j}\right) \mathcal{P}_{F R}^{(c)}\left(T, \epsilon \mid r=\zeta_{j}\right)+\left(1-A_{F R}^{(c)}\left(T, \epsilon \mid r=\zeta_{j}\right)\right) \mathcal{P}_{F R}^{(e)}\left(T, \epsilon \mid r=\zeta_{j}\right)\right]
\end{aligned}
$$


Using the results in Section 3.2, we obtained:

In the case of Strict FR.

$$
\begin{aligned}
\mathcal{P}_{\text {Str }}^{(c)}(T, \epsilon)=\int_{0}^{\infty} & v(T+\hat{T}) \mathscr{L}\left(s_{1}, s_{2}, \lambda\right) d r \\
& +\int_{0}^{\infty} v\left(\frac{\hat{T}}{\phi}\right) \mathscr{L}_{I_{\theta}^{(o c)}}\left(s_{2}, \frac{\lambda}{\Delta}\right)\left(1-e^{-\frac{T}{S N R} r^{\alpha(1-\epsilon)}} \mathscr{L}_{I_{\theta}^{(o c)}}\left(s_{1}, \lambda\right)\right) d r
\end{aligned}
$$

In the case of Soft FR.

$$
\begin{aligned}
\mathcal{P}_{\text {Sof }}^{(e)}(T, \epsilon)=\int_{0}^{\infty} v(T+\hat{T}) \mathscr{L}\left(s_{1}, s_{2}, \frac{\Delta-1}{\Delta} \lambda\right) \mathscr{L}\left(\phi s_{1}, \phi s_{2}, \frac{\lambda}{\Delta}\right) d r \\
+\int_{0}^{\infty} v\left(\frac{\hat{T}}{\phi}\right) \mathscr{L}_{I_{\theta}^{(o c)}}\left(\frac{s_{2}}{\phi}, \frac{\Delta-1}{\Delta} \lambda\right) \mathscr{L}_{I_{\theta}^{(o c)}}\left(s_{2}, \frac{1}{\Delta} \lambda\right) \\
\quad \times\left(1-e^{-\frac{T r \alpha(1-\epsilon)}{S N R}} \mathscr{L}_{I_{\theta}^{(o c)}}\left(s_{1}, \frac{\Delta-1}{\Delta} \lambda\right) \mathscr{L}_{I_{\theta}^{(o c)}}\left(\phi s_{1}, \frac{1}{\Delta} \lambda\right)\right)
\end{aligned}
$$

\subsection{Average Data Rate}

\subsubsection{Average User Data Rate}

The average capacity of the user whose received uplink signal is $S I N R$ is given by the Shannon Theorem, i.e, $C=\mathbb{E}[\ln (1+S I N R)]$ where the expectation is taken over the SINR distribution. In the FR network, the CCU experiences a received $\operatorname{SINR}$ at $\operatorname{SINR}(1, \phi)$ during the communication phase if the measured uplink SINR during the establishment phase is $\operatorname{SIN} R^{(o)}(1, r)>T$. Hence, the average capacity of the CCU is obtained by [7]:

$$
\begin{aligned}
C_{F R}^{(c)}(T, 1) & =\mathbb{E}\left(\ln (\operatorname{SINR}(1, \epsilon)+1) \mid S I N R^{(o)}(1, \epsilon)>T\right) \\
& =\int_{0}^{\infty} \mathbb{P}\left(\ln (\operatorname{SINR}(1, \epsilon)+1)>\gamma \mid S I N R^{(o)}(1, \epsilon)>T\right) d \gamma \\
& =\int_{0}^{\infty} \frac{\mathbb{P}\left(S I N R(1, \epsilon)>e^{\gamma}-1, S I N R^{(o)}(1, \epsilon)>T\right)}{\mathbb{P}\left(S I N R^{(o)}(1, \epsilon)>T\right)} d \gamma
\end{aligned}
$$

Employing a change of variable $t=e^{\gamma}-1$, Equation 28 becomes

$$
C_{F R}^{(c)}(T, 1)=\int_{0}^{\infty} \frac{1}{t+1} \frac{\mathbb{P}\left(S I N R(1, \epsilon)>t, S I N R^{(o)}(1, \epsilon)>T\right)}{\mathbb{P}\left(S I N R^{(o)}(1, \epsilon)>T\right)} d t
$$

The second part of the integrand in Equation 30 is the average coverage probability of the CCU with the coverage threshold $t$. Therefore, the average data rate of the CCU is given by

$$
C_{F R}^{(c)}(T, 1)=\int_{0}^{\infty} \frac{1}{t+1} \mathcal{P}_{F R}^{(c)}(T, \epsilon \mid \hat{T}=t) d t
$$


Similarity, the average data rate of the CEU in this case is obtained by [7:

$$
C_{F R}^{(e)}(T, 1)=\int_{0}^{\infty} \frac{1}{t+1} \mathcal{P}_{F R}^{(e)}(T, \epsilon \mid \hat{T}=t) d t
$$

in which $F R=(S t r, S o f)$ correspond to Strict FR and Soft FR.

Using the results in Section 3.2 in (30) and (31), the average capacities of the CCU and CEU under Strict FR and Soft FR can be obtained.

\subsubsection{Average Network Data Rate}

In order to examine the network performance, we assume that the network is allocated N RBs. Under Strict FR, the RBs are separated into $N_{\text {Str }}^{(c)}$ common RBs and $\Delta$ CE RB groups of $\frac{N_{S t r}^{(e)}}{\Delta}$ $195 \mathrm{RBs}$, in which $N_{\text {Str }}^{(c)}+N_{\text {Str }}^{(e)}=N$. Since the each CE RB group is a private RBs with a group of $\Delta$ cells, each BS is allowed to utilise $N_{S t r}^{(c)}$ CC RBs and $\frac{N_{S t r}^{(e)}}{\Delta}$ CE RBs. Under Soft FR, since each BS can transmit on all allocated RBs, each BS is allocated $N_{\text {Sof }}^{(c)}$ CC RBs and $N_{\text {Sof }}^{(e)}$ CE RBs, in which $N_{\text {Sof }}^{(c)}+N_{\text {Sof }}^{(e)}=N$.

Due to the assumption that each user is allocated a RB during a given timeslot, the BS can 200 serve maximums of $N_{\text {Str }}^{(c)}$ CCUs and $\frac{N_{S t r}^{(e)}}{\Delta}$ CEUs in the case of Strict FR and $N_{\text {Sof }}^{(c)}$ CCUs and $N_{\text {Sof }}^{(e)}$ CEUs in the case of Soft FR. Therefore, the average network data rates under Strict FR and Soft FR are given by

- Under Strict FR

$$
C_{S t r}(T)=N_{S t r}^{(c)} C_{S t r}^{(c)}(T, \epsilon)+\frac{N_{S t r}^{(e)}}{\Delta} C_{S t r}^{(e)}(T, \epsilon)
$$

- Under Soft FR

$$
C_{\text {Sof }}(T)=N_{\text {Sof }}^{(c)} C_{\text {Sof }}^{(c)}(T, \epsilon)+N_{\text {Sof }}^{(e)} C_{\text {Sof }}^{(e)}(T, \epsilon)
$$

in which $C_{F R}^{(c)}$ and $C_{F R}^{(e)}$ are average data rates of the CUU and CEU and defined in 30 and 31 .

\section{Simulation and Discussion}

In this section, we present numerical and simulation results to verify analytical results and the relationship between the SINR threshold and power control exponent on the network performance. The analytical parameters are based on 3GPP recommendations [24] such as path loss exponent $\alpha=3.5$ and $P=-76 \mathrm{dBm}$ and $\sigma^{2}=-99 \mathrm{dBm}$.

Since the numerical results of the exact expressions in forms of integrals are perfectly equal these of the corresponding approximated expressions, a term analytical results is used to represent 
the numerical results of these expressions.

\subsection{Validation of the Analytical Results}

The analytical results in Section 3.2 are compared with the Monte Carlo simulation. As shown in Fig. 1 the lines representing the analytical results perfectly match with the star points representing

in SINR and user's performance. Consequently, it is observed from Fig. 1 1 that the user achieves a higher performance when the signals including serving and interfering signals experience pathloss with a higher pathloss exponent.

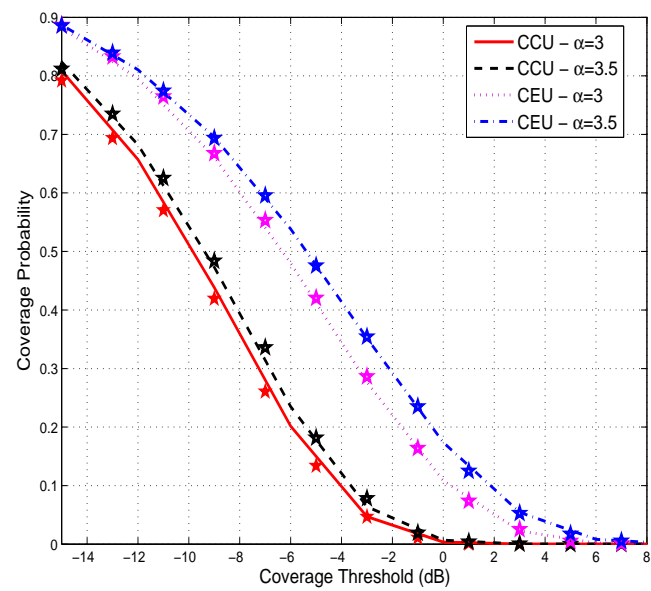

(a) Strict FR

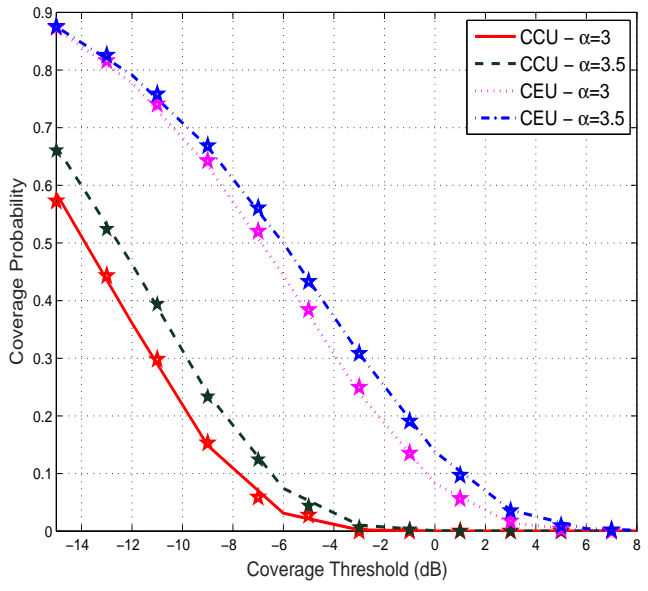

(b) Soft FR

Figure 1: Comparison the analytical results and Monte Carlo simulation lines: theoretical results, stars: simulation results

As shown in Fig. 1. the Strict FR outperforms Soft FR in term of average probability for both $\mathrm{CCU}$ and $\mathrm{CEU}$. In the case of $\mathrm{CCU}$, the CCU under Strict FR experiences interference, which is generated by the CCUs while under Soft FR, each CCU is affected by interference from both CCUs and CEUs. Hence, the CCU under Soft FR experiences higher interference and achieves lower performance than that under Strict FR. For example, when coverage threshold $\hat{T}=-9 \mathrm{~dB}$ and $\alpha=3.5$, the average coverage probability of the CCU under Strict FR is 0.4839 , which $18 \%$ greater than that under Soft FR.

In the case of CEU, the CEU under Strict FR experiences interference from other CEUs, but the density of interfering users is only $\frac{1}{\Delta} \lambda$. Meanwhile, the CEU under Soft FR is affected by the CCUs with density $\frac{\Delta-1}{\Delta} \lambda$ and the CEUs with density $\frac{1}{\Delta} \lambda$. Hence, the CEU under Strict FR 
experiences lower interference and consequently outperforms the CEU under Soft FR. However, as shown in Fig. 1b the difference between performances of the CEU under Strict FR and Soft FR are not significant.

\subsection{Effects of the Density of BSs}

When the density of BSs increases which means more BSs are deployed in the network, the distances between the user and BSs reduces, which leads to a decline in pathloss of both serving and interfering signals. However, the improvement of the serving signal overcomes an increase in interference [25, then the uplink SINR increases with the density of BSs. Therefore, it can be observed in Fig. 2 that for both Strict FR and Soft FR, the number of CCUs whose transmit powers are $\phi$ times less than CEUs increases with the density of BSs. Furthermore, when the density of BSs $\lambda$ increases, the distance from the user to the tagged BS and consequently the user transmit power reduce. As a result, the average transmit power of the typical user, which can be served as a CCU or CEU reduces.
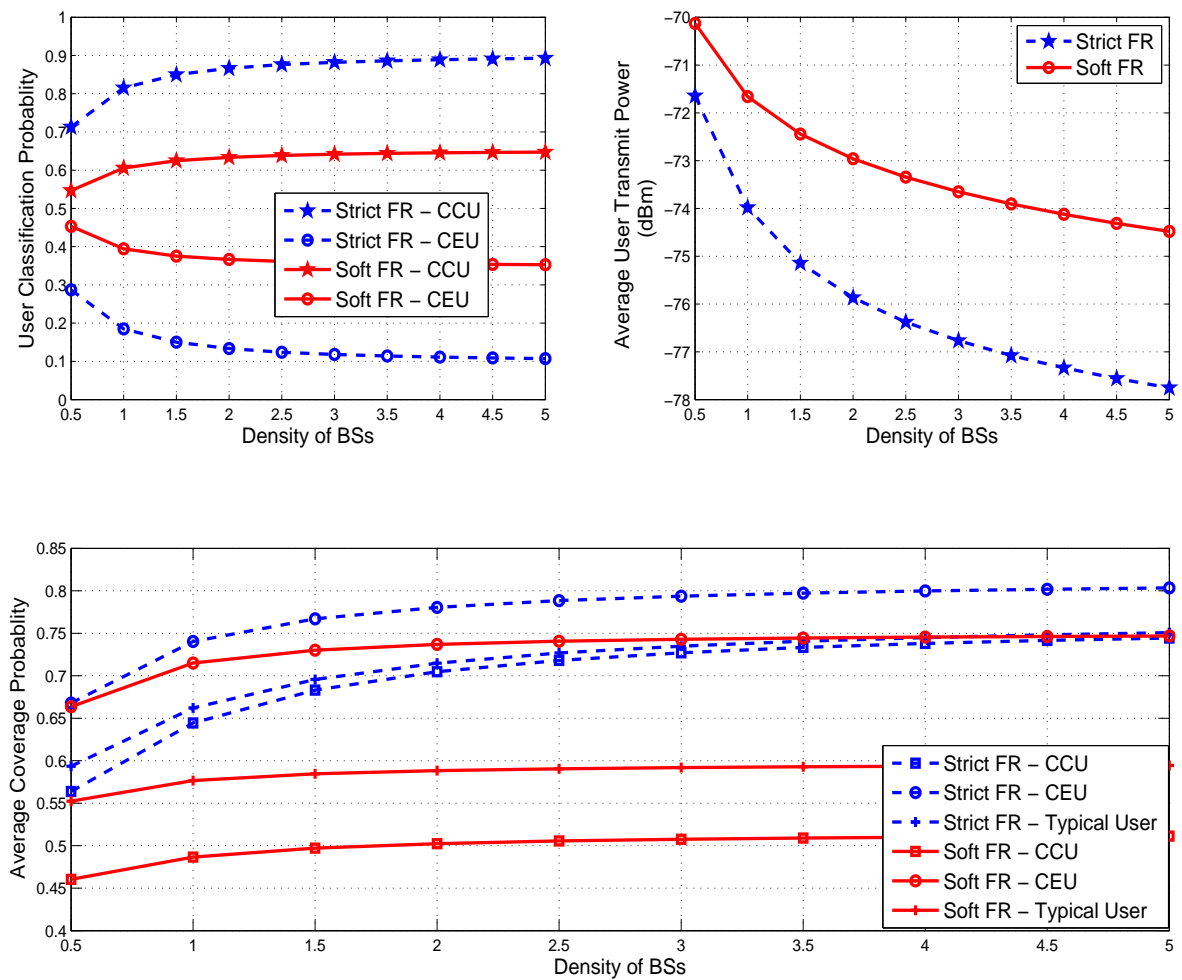

Figure 2: Effects of BSs' Density on the Network Performance

Interestingly, although the average transmit power reduces, the average coverage probabilities of both CCU and CEU increase. Take Strict FR for example, when $\lambda$ increases from $1 B S / \mathrm{km}^{2}$ to $3 \mathrm{BS} / \mathrm{km}^{2}$, the user transmit power reduces by $2.79 \mathrm{dBm}$ from $-73.98 \mathrm{dBm}$ to $-76.77 \mathrm{dBm}$ but the average coverage probabilities increase by $14.03 \%$ from 0.6445 to 0.7349 in the case of CCU and $4.0 \%$ from 0.7149 to 0.7429 in the case of CEU. Therefore, increasing the number of BSs in 
the network can be considered as the approach to save user's power consumption of the user as well as improve network performance.

As discussion in Section 4.1, since both CCU and CEU under Soft FR are more strongly affected by interference than those under Strict FR, more users under Soft FR are served as CEUs than under Strict FR. Therefore, the typical user under Soft FR consumes more energy than that under Strict FR. For example, when the BSs are distributed with a density of $1 \mathrm{BS} / \mathrm{km}^{2}$, the CCU classification probabilities are $18.50 \%$ in the case of Strict FR and $39.44 \%$ in the case of Soft FR. In addition, the typical user under Soft FR transmits at $-71.66 \mathrm{dBm}$ on average, which is 2.3 $d B m$ (approximately 1.706 times) greater than that under Strict FR.

\subsection{Effects of the Power Control Exponent}

To evaluate the effect of the power control exponent $\epsilon$ on the user's performance, we consider different network scenarios from sparse to dense networks [26]. Since, the average transmit power of the CEU is obtained by multiplying the average transmit power of the CCU by $\phi$ in which $\phi$ is

265 a constant number, changes in downward and upward trends of the average CCU and CEU powers are the same. Hence, in this section, we investigate the average transmit power of CCU. Since the average transmit power of the $\mathrm{CCU}$ only depends on the distance from the user to it's serving BS and the path loss exponent, the average CCU transmit powers are the same for both Strict FR and Soft FR. Fig. 3 and 4 shows that the user's transmit power increases in the case of $\lambda=0.1$ 270 $B S / \mathrm{km}^{2}$, reduces in the case of $\lambda=1 \mathrm{BS} / \mathrm{km}^{2}$ but a decline followed by an increase in the case of $\lambda=0.5 B S / \mathrm{km}^{2}$. This finding contradicts the conclusion for $\lambda=0.24 B S / \mathrm{km}^{2}$ in [21, which stated that the average transmit power of the users greatly reduces when an increase in $\epsilon$.

\section{For a sparse network such as in rural area}

The cell radius is usually around $10 \mathrm{~km}$ and the density of BSs is approximately $\lambda \approx 0.1$ $275 B S / \mathrm{km}^{2}$. The average distance from the user to the serving BS is $E[r]=\int_{0}^{\infty} 2 \pi \lambda r^{2} e^{-\pi \lambda r^{2}}=1.581$ $\mathrm{km}$. It is obviously that there exist users with the distance to the serving BSs $r<1 \mathrm{~km}$ and others with $r>1$. When $\epsilon$ increases, the transmits powers drop rapidly for the users having distances $r<1 \mathrm{~km}$ and exponentially increase for the users having distances $r>1$. Since the average distance is $1.581 \mathrm{~km}$, the number of users with $r>1 \mathrm{~km}$ is much greater than those with $r<1$ $\mathrm{km}$. Therefore, it is clear that the average transmit power of the typical user increases with $\epsilon$.

It is very interesting that in the case of Strict FR, although the average uplink SINR on the $\mathrm{CC} \mathrm{RB}$ during the establishment phase improves with the power control exponent $\epsilon$, which is represented through an increase in the number of CCUs, the average coverage probability of the CCU passes a rapid decline as shown in Fig. The phenomenon can be explained as the following 285 hypothesis: when $\epsilon$ increases, the interference from the users with $r>1 \mathrm{~km}$ increases while that 

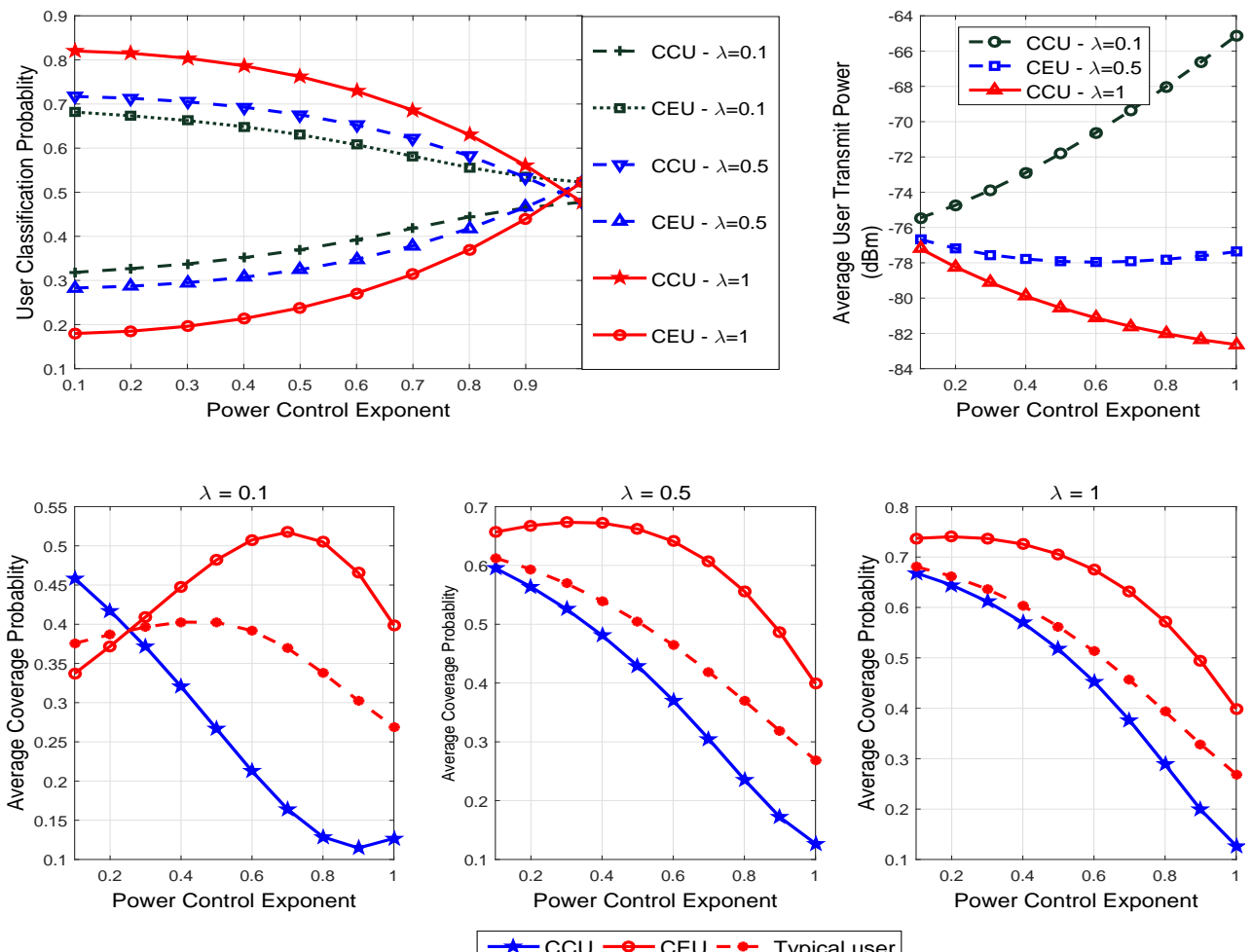

Figure 3: (Strict FR) Effects of the Power Control Exponent on the Network Performance
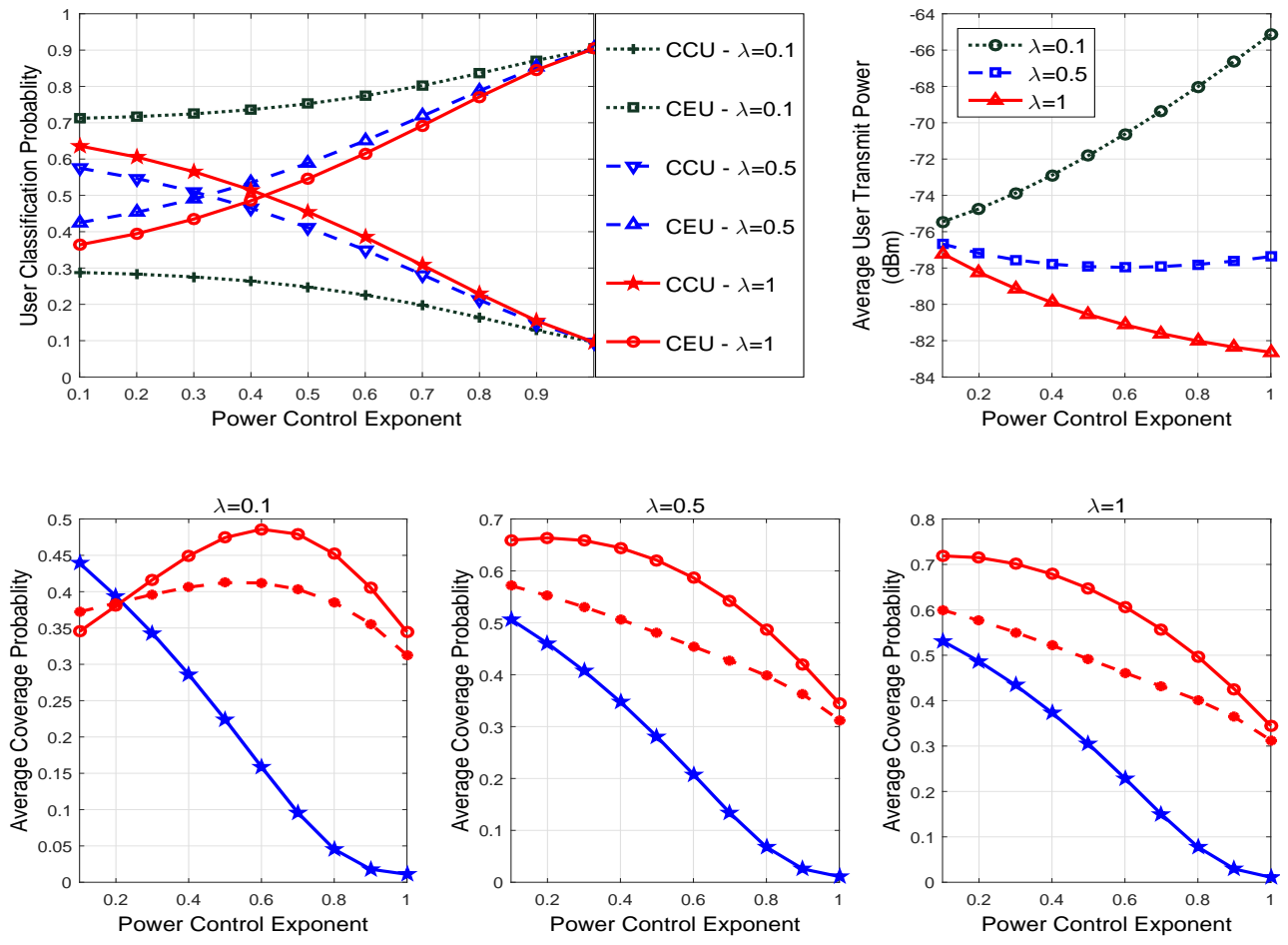

Figure 4: (Soft FR) Effects of the Power Control Exponent on the Network Performance

from the users with $r<1 \mathrm{~km}$ reduces. Since in this scenario, the number of users with $r>1$ $\mathrm{km}$ is much greater than those with $r<1 \mathrm{~km}$, the total uplink interference can increases with $\epsilon$ though all interfering users transmit at the same power. Thus, the users with $r<1 \mathrm{~km}$ experience 
lower uplink SINRs but most of these users are still defined as the CCUs due to a very small value of SINR threshold, e.g $T=-10 \mathrm{~dB}$, while the users with $r>1 \mathrm{~km}$ may achieve higher uplink SINR and may be classified as the CCUs. Hence, it is obviously that more users are classified as the $\mathrm{CCU}$ in this case. In other words, the CCU classification probability during the establishment phase increases with $\epsilon$.

During the communication phase, in the case of the CCU who is usually close the serving BS Thus, the uplink SINRs of the CCUs decrease rapidly, which is represented in a fall trend of average coverage probability as shown in Fig. 3. In the case of the CEU who is usually far from the serving BS, the CEU transmit power significantly increases with $\epsilon$, which can overcome the rise of interference. Hence, the CEU may achieve higher performance when $\epsilon$ increases. However, at a high value of $\epsilon$ such as $\epsilon=0.7$, the increase in the CEU transmit power can not trade off with the growth of interference, which results in a reduction of the average coverage probability.

In the case of Soft FR, since the CC RB experiences interference from both CCUs and CEUs who are usually farther from the serving BSs (conventionally $r>1 \mathrm{~km}$ ), than CCUs and transmit at a high power, which is $\phi=10$ times greater than the transmit power of the CCUs. When $\epsilon$ increases, the interference from the CEUs increases with a higher rate than the reduction in interference from the CCUs. Therefore, the interference on the CC RB increases with $\epsilon$, which causes of a drop in uplink SINR of the users, especially for the users with $r<1 \mathrm{~km}$. Therefore, in this case, more users are served as the CEUs when $\epsilon$ increases, which is presented through the upward trend of the CEU classification probability in Fig. 4

It is remarked that when the user is classified as the CEU on the uplink, it will transmit at a high transmit power while the interference is unlikely to change. Therefore, pushing more users to be CEUs, especially the users have the received SINRs during the establishment phase are around the SINR threshold, can improve the average uplink SINR and then the average coverage probability of the CEU. However, when $\epsilon$ increases to a high value such as $\epsilon=0.7$ in the case of Soft FR, the interference from the CEUs may be much more greater than that from the CCUs and the Gaussian

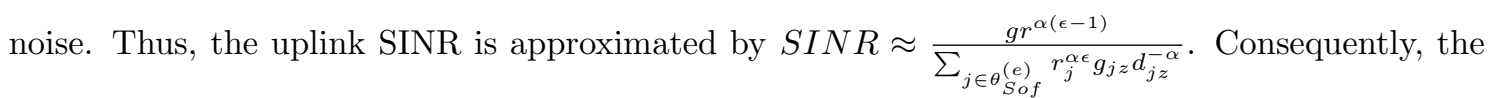
use of the high transmit power to serve user in this case may not bring any benefit to the user performance. As a result, the average coverage probability in this case is smaller than that in the case of a low value of $\epsilon$. Furthermore, the optimal values of the power control exponent can be selected at $\epsilon=0.7$ in the case of Strict FR and $\epsilon=0.6$ in the case of Soft FR where the average coverage probabilities of the typical user are at the peaks of 5.2 and 0.48 respectively. 
For a medium dense network such as in urban

The cell radius usually fluctuates from $1-2 \mathrm{~km}$ and the density of BSs is around $0.5 \mathrm{BS} / \mathrm{km}^{2}$. The average distance in this case is $E[r]=0.7071 \mathrm{~km}$. Hence the transmit power of both a CCU $\operatorname{Pr}^{\epsilon \alpha}$ and a CEU $\phi \operatorname{Pr}^{\epsilon \alpha}$ reduces when $\epsilon$ increases. However, since the CCU classification probability increases with $\epsilon$, the average transmit power of the typical user slowly reduces to the bottom at $-77.92 \mathrm{dBm}$ when $\epsilon=0.7$ and before marginally increasing. As shown in the figure, the average coverage probability of the user reduces very quickly since high values of $\epsilon$ lead to an increase in the transmit power from interfering users having great path loss, and growth of interference at the BS.

For a dense network such as in the city center

The BSs may be distributed every kilometre, and thus the density of BSs is $\lambda=1 \mathrm{BS} / \mathrm{km}^{2}$. In this case, the user is usually very close it's serving BS with an average distance of $E[r]=0.5$ $k m$. Hence, both the average transmit power of the CCU and CEU reduces when $\epsilon$ increase. 335 However, the trend of the coverage probability on average is similar in the case of the urban network. Therefore, the optimal values of $\epsilon$ in this case can be chosen at $\epsilon=0$, in which the user's performance is at the maximum value.

\subsection{Average Network Data Rate Comparison}

In this section, the average data rates of the networks using Strict FR and Soft FR are compared as shown in Fig. 5. It is assumed that the network is allocated $75 \mathrm{RBs}$, which corresponds to 15 MHz. As discussion in Section 3.4.2 the number of CC and CE RBs under Strict FR are $N_{\text {Str }}^{(c)}=30$ and $N_{\text {Str }}^{(e)}$ in the case of Strict FR, and $N_{\text {Sof }}^{(c)}=50$ and $N_{\text {Sof }}^{(e)}=25$ in the case of Soft FR.

It is obvious that the CEU classification probability significantly increases with the SINR threshold. As discussion in previous sections, the uplink in the case of Strict usually achieves higher SINR than that in the case of Soft FR. In other words, more users under Strict FR achieve higher uplink SINRs than under Soft FR. Hence, most users under Soft FR are classified as CEUs even when SINR threhold is at a low value. Consequently, when SINR threshold increases to a high value, more users in the Strict FR are being classified as new CEUs. Therefore, although the user under Soft FR can be classified as the CEU with a higher probability than that under Strict FR, the probability of CEU classification under Strict FR increases at a higher rate than that under Strict FR. For example, when the SINR threshold increases from $-4 \mathrm{~dB}$ to $0 \mathrm{~dB}$, the rate under Strict FR is 0.294 while that under Soft FR is 0.253 .

In contradiction to the conclusion in Section 4.1 that stated that the Strict FR outperforms Soft FR in term of average coverage probability of both CCU and CEU, Fig. 5 5 indicates that Soft 355 FR achieves significantly higher average cell data rate, which is the sum of average data rate of 

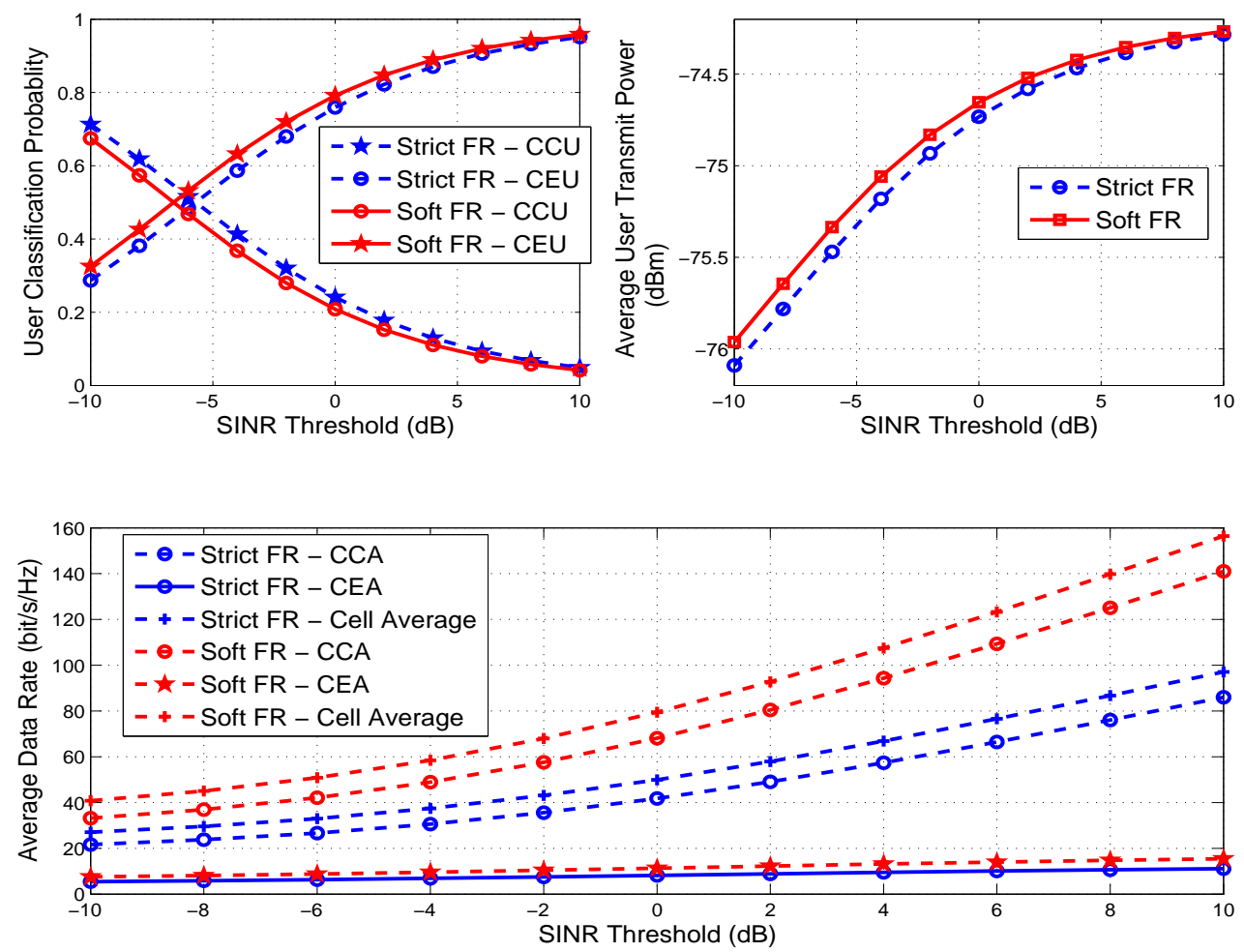

Figure 5: Effects of the SINR threshold on average cell data rate

all users within a cell, than Strict FR. Take SINR threshold $T=0$ for example, the average data rate of the network using Soft FR is approximately $79.48(\mathrm{bit} / \mathrm{s} / \mathrm{Hz})$, which is $58.96 \%$ greater than that using Strict FR. This is due to the fact that although Soft FR can create more interference than Strict FR, each cell in the network using Soft FR allows to reuse all RBs, i.e. $N_{c}+N_{e}$, while under Strict FR, each cell is only allowed to resue $N_{c}+\frac{N_{e}}{\Delta}$ RBs and thus more users can be served at the same time than Strict FR.

\section{Conclusion}

In this paper, we modelled the uplink of the cellular network - PPP model using Strict FR and Soft FR algorithms, which is recommended by 3GPP. The analytical results which are verified by the Monte Carlo simulation focus on the network performance parameters such as the CCU and CEU classification probabilities, the average transmit power, and average coverage probability. The close-form expressions of the performance indexes are derived by using Gaussian Quadrature. While the Strict FR outperforms Soft FR in terms of average coverage probability and power consumption of both CCU and CEU, Soft FR can achieve the higher cell data rate. For both

370 Strict FR and Soft FR, the user can achieve higher performance and consumes lower power when the density of BSs increases. While for a medium dense network with $\lambda=0.5 B S / \mathrm{km}^{2}$ and a dense network with $\lambda=1 B S / \mathrm{km}^{2}$, the user's performance is at the maximum value when all users transmit at their constant powers, e.g. $P$ for CCUs and $\phi P$ for CEUs. Meanwhile, for a 
sparse network with $\lambda=0.1 \mathrm{BS} / \mathrm{km}^{2}$, the average coverage probability of the CEU during the communication phase increases significantly to the peak before undergoing a rapid decline when the power control exponent increases. This finding is very interesting and has not been found in previous works.

\section{Appendix A. (Soft FR): The probability in which the user is served as a CCU}

The probability, in which the user is served as a CCU, is given by

$$
A_{\text {Str }}^{(c)}(T, \epsilon \mid r)=\mathbb{P}\left(\frac{P g r^{\alpha(\epsilon-1)}}{\sigma^{2}+I_{\text {Sof }}^{(c)}(\phi)}>T\right)
$$

in which $I_{\text {Sof }}^{(c)}(\phi)$ is defined in Equation 4 .

Since $g$ has a exponential distribution, we have

$$
\begin{aligned}
A_{S t r}^{(c)}(T, \epsilon \mid r) & =e^{-\frac{T r^{\alpha(1-\epsilon)}}{S N R}} \mathbb{E}\left[e^{-\frac{T}{P r^{\alpha(\epsilon-1)}}\left[\sum_{j \in \theta_{\text {Sof }}^{(c)}} P_{j}^{(c)} g_{j z} d_{j z}^{-\alpha}+\sum_{j \in \theta_{S o f}^{(e)}} P_{j}^{(e)} g_{j z} d_{j z}^{-\alpha}\right]}\right] \\
& \stackrel{(\mathrm{a})}{=} e^{-\frac{T r^{\alpha(1-\epsilon)}}{S N R}} \prod_{j \in \theta_{\text {Sof }}^{(c)}} \mathbb{E}\left[e^{-s^{\prime} r_{j}^{\alpha \epsilon} d_{j z}^{-\alpha} g_{j z}}\right] \prod_{j \in \theta_{S o f}^{(e)}} \mathbb{E}\left[e^{-\phi s^{\prime} r_{j}^{\alpha \epsilon} d_{j z}^{-\alpha} g_{j z}}\right] \\
& \stackrel{(\mathrm{b})}{=} e^{-\frac{T r^{\alpha(1-\epsilon)}}{S N R}} \prod_{j \in \theta_{\text {Sof }}^{(c)}} \mathbb{E}\left[\frac{1}{1+s^{\prime} r_{j}^{\alpha \epsilon} d_{j z}^{-\alpha}}\right] \prod_{j \in \theta_{S o f}^{(e)}} \mathbb{E}\left[\frac{1}{1+\phi s^{\prime} r_{j}^{\alpha \epsilon} d_{j z}^{-\alpha}}\right]
\end{aligned}
$$

380 in which $(a)$ due to the independence of $\theta_{\text {Sof }}^{(c)}$ and $\theta_{\text {Sof }}^{(e)}$, and $s^{\prime}=\operatorname{Tr}^{\alpha(1-\epsilon)} ;(b)$ follows the assumption that the channel power gain has an exponential distribution.

Since $r_{j}$ is the distance from user $j$ to it's serving BS, the PDF of $r_{j}$ follows (1). Taking the expectation on $r_{j}$, we obtain

$$
A_{S t r}^{(c)}(T, \epsilon \mid r)=e^{-\frac{T r^{\alpha}(1-\epsilon)}{S N R}} \prod_{j \in \theta_{\text {Sof }}^{(c)}} \mathbb{E}\left[\int_{0}^{\infty} \frac{\frac{2 \pi(\Delta-1) \lambda}{\Delta} t e^{-\frac{\pi(\Delta-1) \lambda}{\Delta}} t^{2}}{1+s^{\prime} t^{\alpha \epsilon} d_{j z}^{-\alpha}} d t\right] \prod_{j \in \theta_{\text {Sof }}^{(e)}} \mathbb{E}\left[\int_{0}^{\infty} \frac{\frac{2 \pi \lambda t}{\Delta} e^{-\frac{\pi \lambda}{\Delta} t^{2}}}{1+\phi s^{\prime} t^{\alpha \epsilon} d_{j z}^{-\alpha}} d t\right]
$$

Given that the density of users in $\theta_{\text {Sof }}^{(c)}$ and $\theta_{\text {Sof }}^{(e)}$ are $\frac{\Delta-1}{\Delta} \lambda$ and $\frac{1}{\Delta} \lambda$. Hence, using the properties of Probability Generating Function (PGF),

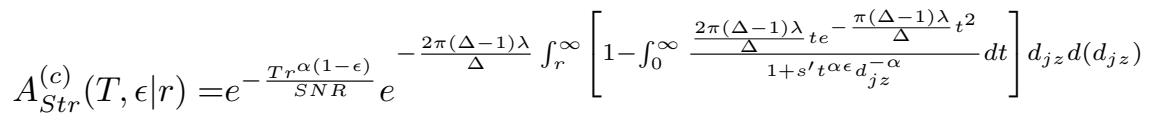

$$
\begin{aligned}
& e^{-\frac{2 \pi \lambda}{\Delta} \int_{r}^{\infty}\left[1-\int_{0}^{\infty} \frac{\frac{2 \pi \lambda t}{\Delta} e^{-\frac{\pi \lambda}{\Delta} t^{2}}}{1+\phi s^{\prime} t^{\alpha \epsilon} d_{j z}^{-\alpha}} d t\right] d_{j z} d\left(d_{j z}\right)}
\end{aligned}
$$


By letting $s_{1}=T r^{\alpha \epsilon}$, and using the change of variable $x=\frac{d_{j z}}{r}$,

$$
A_{S t r}^{(c)}(T, \epsilon \mid r)=e^{-\frac{T r^{\alpha(1-\epsilon)}}{S N R}} \mathscr{L}_{I_{\theta}^{(o c)}}\left(s_{1}, \frac{\Delta-1}{\Delta} \lambda\right) \mathscr{L}_{I_{\theta}^{(o c)}}\left(\phi s_{1}, \frac{1}{\Delta} \lambda\right)
$$

The Remark 2.2 is proved.

\section{Appendix B. Approximate Results in Remark 2.1 and 2.2}

In order to approximated the results in Remark 2.1 and 2.2. Gauss - Laguerre and Gauss Legendre Quadrature are utilised, in which Gauss - Laguerre Quadrature approximate infinite integrals where the integrand has an exponential element [27.

$$
\int_{0}^{\infty} f(t) e^{-t} \approx \sum_{i=1}^{N_{G L}} \omega_{i} f\left(t_{i}\right)
$$

and Gauss - Legendre Quadrature can approximate the definite integral, which conventionally taken over $[a, b]$

$$
\int_{a}^{b} f(x) d x \approx \frac{b-a}{2} \sum_{m=1}^{N_{G}} c_{i} f\left(\frac{a-b}{2} x_{i}+\frac{a+b}{2}\right)
$$

where $N_{G L}$ and $N_{G}$ are the degree of the Laguerre and Legendre polynomial, $t_{i}$ and $w_{i}, c_{i}$ and $x_{i}$ are the $i$-th node and weight, abscissas and weight of the corresponding quadratures.

Approximate $\mathscr{L}_{I_{\theta}^{(o c)}}(s, \lambda)$. Due to the fact that $\int_{0}^{\infty} \pi \lambda t e^{-\pi \lambda t^{2}} d t=1$, the integral in $\mathscr{L}_{I_{\theta}^{(o c)}}(s)$, denoted by $f(s, \lambda)$, can be obtained by

$$
f(s, \lambda)=\int_{0}^{\infty} \pi \lambda t^{\alpha \epsilon} t e^{-\pi \lambda t^{2}} \int_{1}^{\infty} \frac{s x^{1-\alpha}}{1+s t^{\alpha \epsilon} x^{-\alpha}} d x d t
$$

Employing the change of variable $\gamma=s x^{-\alpha} t^{\alpha \epsilon}$, then $f(s, \lambda)=$

$$
\int_{0}^{\infty} \frac{\pi \lambda s^{\frac{2}{\alpha}}}{\alpha} t^{\epsilon} t e^{-\pi \lambda t^{2}} \int_{0}^{\infty} \frac{\gamma^{-2 / \alpha}}{1+\gamma} d \gamma d t-\int_{0}^{\infty} \frac{\pi \lambda s}{2} t^{\alpha \epsilon} t e^{-\pi \lambda t^{2}} \int_{0}^{1} \frac{1}{x^{\frac{\alpha}{2}}+s t^{\alpha \epsilon}} d x d t
$$

In case of $\alpha>2$, according to the properties of Gamma function [27], $\int_{0}^{\infty} \frac{\gamma^{-2 / \alpha}}{1+\gamma} d \gamma=\frac{\pi}{\sin \left(\frac{2 \pi}{\alpha}\right)}$. Employing Gauss - Legendre approximation for $\int_{0}^{1} \frac{1}{x^{\frac{\alpha}{2}}+s t^{\alpha \epsilon}} d x$, thus

$$
f(s, \lambda) \approx \frac{\pi s^{\frac{2}{\alpha}}}{\alpha \sin \left(\frac{2 \pi}{\alpha}\right)} \int_{0}^{\infty} t^{\epsilon} \pi \lambda t e^{-\pi \lambda t^{2}} d t-\frac{s}{4} \int_{0}^{\infty} \sum_{i=0}^{N_{G}} \frac{c_{i} t^{\alpha \epsilon} \pi \lambda t e^{-\pi \lambda t^{2}}}{\eta_{i}^{\frac{\alpha}{2}}+s t^{\alpha \epsilon}} d t
$$

in which $\eta_{i}=\frac{x_{i}+1}{2}$. 
Using a change of variable $\zeta=\pi \lambda t^{2}$, the integral has a suitable form of Gauss - Laguerre Quadrature. Hence, $f(s, \lambda)$ is approximated by

$$
f(s, \lambda) \approx \frac{\pi s^{\frac{2}{\alpha}}}{\alpha \sin \left(\frac{2 \pi}{\alpha}\right)} \sum_{m=0}^{N_{G L}} w_{m} \zeta_{m}^{\epsilon}-\frac{s}{4} \sum_{j=0}^{N_{G L}} w_{j} \zeta_{m}^{\alpha \epsilon} \sum_{i=0}^{N_{G}} \frac{c_{i}}{\eta_{i}^{\frac{\alpha}{2}}+s \zeta_{m}^{\frac{\alpha \epsilon}{2}}}
$$

in which $\zeta_{m}=\sqrt{\frac{t_{m}}{\pi \lambda}}\left(\forall 1 \leq m \leq N_{G L}\right)$.

Approximate $A_{S t r}^{(c)}(T, \epsilon)$. Employing a change of variable $t=\pi \lambda r^{2}$, then $A_{S t r}^{(c)}(T, \epsilon)$ equals

$$
A_{S t r}^{(c)}(T, \epsilon)=\int_{0}^{\infty} e^{-t-\frac{T}{S N R}\left(\frac{t}{\pi \lambda}\right)^{\alpha(1-\epsilon) / 2}} \mathscr{L}_{I_{\theta}^{(o c)}}\left(T(t / \pi \lambda)^{-\alpha \epsilon / 2}, \lambda\right) d \zeta
$$

Using the Gauss - Laguerre Quadrature, then $A_{S t r}^{(c)}(T, \epsilon)$ is approximately obtained by

$$
A_{S t r}^{(c)}(T, \epsilon) \approx \sum_{j=0}^{N_{G L}} e^{-\frac{T}{S N R} \zeta_{j}^{\alpha(1-\epsilon)}} \mathscr{L}_{I_{\theta}^{(o c)}}^{(j)}\left(T \zeta_{j}^{-\alpha \epsilon}, \lambda\right)
$$

in which $\mathscr{L}_{I_{\theta}^{(o c)}}^{(j)}(s, \lambda) \approx e^{\pi \lambda \zeta_{j}^{2}\left[\frac{2 \pi s \frac{2}{\alpha}}{\alpha \sin \left(\frac{2 \pi}{\alpha}\right)} \sum_{m=0}^{N_{G L}} w_{j} \zeta_{m}^{\epsilon}-\frac{s}{2} \sum_{m=0}^{N_{G L}} w_{m} \zeta_{m}^{\alpha \epsilon} \sum_{i=0}^{N_{G}} \frac{c_{i}}{\eta_{i}^{\frac{\alpha}{2}}+s \zeta_{m}^{\frac{\alpha \epsilon}{2}}}\right]}$

Approximate $A_{\text {Sof }}^{(c)}(T, \epsilon)$. Similarity, $A_{\text {Sof }}^{(c)}(T, \epsilon)$ is approximated by

$$
\sum_{j=0}^{N_{G L}} e^{-\frac{T}{S N R} \zeta_{j}^{(1-\epsilon)}} \mathscr{L}_{I_{\theta}^{(o c)}}^{(j)}\left(T \zeta_{j}^{-\alpha \epsilon}, \frac{\Delta-1}{\Delta} \lambda\right) \mathscr{L}_{I_{\theta}^{(o c)}}^{(j)}\left(\phi T \zeta_{j}^{-\alpha \epsilon}, \frac{1}{\Delta} \lambda\right)
$$

The proposition is proved.

\section{Appendix C. (Strict FR) The Average Coverage Probability of CCU}

The coverage probability of a CCU under the Strict FR network is obtained by

$$
\begin{aligned}
\mathcal{P}_{c}^{(c)}(T, \epsilon)= & \frac{\mathbb{P}\left(\frac{P^{(c) g r^{-\alpha}}}{\sigma^{2}+I_{S t r}^{(c)}(\phi)}>\hat{T}, \frac{P^{(c)} g^{(o)} r^{-\alpha}}{\sigma^{2}+I_{S t r}^{(o c)}(\phi)}>T\right)}{\mathbb{P}\left(\frac{P g r \alpha(\epsilon-1)}{\sigma^{2}+I_{S t r}^{(o c)}(\phi)}>T\right)} \\
= & \frac{\int_{0}^{\infty} r e^{-\pi \lambda r^{2}} e^{-\frac{(T+\hat{T}) \sigma^{2}}{P(c) r-\alpha}} \mathbb{E}\left[e^{-\frac{\hat{T}^{(c)}(c)}{P_{S t r}(c)-\alpha}-\frac{T I_{S t r}^{(o c)}}{P^{(c)} r-\alpha}}\right] d r}{\int_{0}^{\infty} r e^{-\pi \lambda r^{2}}\left(e^{-\frac{T \sigma^{2}}{\left.P^{(c)}\right)^{-\alpha}}} \mathbb{E}\left[-\frac{T I_{S t r}^{(o c)}}{P^{(c)} r^{-\alpha}} d r\right]\right) d r}
\end{aligned}
$$

The expectation in the numerator of C.1 is the joint Laplace transform of interference during the establishment $I_{S t r}^{(o c)}$ and communication phase $I_{S t r}^{(c)}$, denoted by $\mathscr{L}\left(s_{1}^{\prime}, s_{2}^{\prime}, \lambda\right)$ and joint evaluated at 
$s_{1}^{\prime}=\operatorname{Tr}^{\alpha(1-\epsilon)}$ and $s_{2}^{\prime}=\hat{T} r^{\alpha(1-\epsilon)}$

$$
\begin{aligned}
\mathscr{L}\left(s_{1}^{\prime}, s_{2}^{\prime}, \lambda\right) & =\mathbb{E}\left[e^{-s_{1}^{\prime} \sum_{j \in \theta} r_{j}^{\alpha \epsilon} g_{j z} d_{j z}^{-\alpha}-s_{2}^{\prime} \sum_{j \in \theta_{S t r}^{(c)}} r_{j}^{\alpha \epsilon} g_{j z}^{(o)} d_{j z}^{-\alpha}}\right] \\
& =\prod_{j \in \theta} \mathbb{E}\left[\frac{1}{1+s_{1}^{\prime} r_{j}^{\alpha \epsilon} d_{j z}^{-\alpha}} \frac{1}{1+s_{2}^{\prime} r_{j}^{\alpha \epsilon} d_{j z}^{-\alpha}}\right]
\end{aligned}
$$

in which (C.2) due to the assumption that all channel gains are independent Rayleigh fading.

Since $r_{j}$ is the distance from user $j$ to it's serving BS, the PDF of $r_{j}$ follows (1). Taking the expectation on $r_{j}$, we obtain

$$
=\prod_{j \in \theta} \mathbb{E}\left[\int_{0}^{\infty} \frac{2 \pi \lambda t e^{-\pi \lambda t^{2}}}{\left(1+s_{1}^{\prime} t^{\alpha \epsilon} d_{j z}^{-\alpha}\right)\left(1+s_{2}^{\prime} t^{\alpha \epsilon} d_{j z}^{-\alpha}\right)} d t\right]
$$

Given that $d_{j z}$ is the distance from the interfering user $j$ to the serving BS of user $z$ and the density of the interfering users is as same as the BSs' density, using the properties of Probability Generating Function (PGF), Equation C.3 becomes

$$
=e^{-2 \pi \lambda \int_{r}^{\infty}\left[1-\int_{0}^{\infty} \frac{2 \pi \lambda t e^{-\pi \lambda t^{2}}}{\left(1+s_{1}^{\prime} t^{\alpha \epsilon} d_{j z}^{-\alpha}\right)\left(1+s_{2}^{\prime} t^{\alpha \epsilon} d_{j z}^{-\alpha}\right)} d t\right] d_{j z} d\left(d_{j z}\right)}
$$

By letting $s_{1}=T r^{\alpha \epsilon}$ and $s_{2}=\hat{T} r^{\alpha \epsilon}$, and using the change of variable $x=\frac{d_{j z}}{r}$, the joint Laplace transform

$$
\mathscr{L}\left(s_{1}, s_{2}, \lambda\right)=e^{-2 \pi \lambda r^{2} \int_{1}^{\infty}\left[1-\int_{0}^{\infty} \frac{2 \pi \lambda t e^{-\pi \lambda t^{2}}}{\left(1+s_{1} t^{\alpha \epsilon_{x}-\alpha}\right)\left(1+s_{2} t^{\alpha \epsilon_{x}-\alpha}\right)} d t\right] x d x}
$$

By substituting (9) and (C.5) into (C.1), the Theorem is proved.

\section{Appendix D. (Strict FR) Approximate The Average Coverage Probability of the $\mathrm{CCU}$}

Approximate $\mathscr{L}\left(s_{1}, s_{2}, \lambda\right)$. Since $\int_{0}^{\infty} \pi \lambda t e^{-\pi \lambda t^{2}} d t=1$ and denote $v\left(s_{1}, s_{2}, \lambda\right)$ as the integral of the exponent in the joint Laplace transform, we have

$$
v\left(s_{1}, s_{2}, \lambda\right)=\int_{0}^{\infty} \pi \lambda t e^{-\pi \lambda t^{2}} \int_{1}^{\infty} \frac{\left(s_{1}+s_{2}+s_{1} s_{2} t^{\alpha \epsilon} x^{-\frac{\alpha}{2}}\right) t^{\alpha \epsilon} x^{-\frac{\alpha}{2}}}{\left(1+s_{1} t^{\alpha \epsilon} x^{-\frac{\alpha}{2}}\right)\left(1+s_{2} t^{\alpha \epsilon} x^{-\frac{\alpha}{2}}\right)} d x d t
$$

The inner integral can be presented as the result of the abstraction between into two integrals $I_{0}(t)$ and $I_{1}(t)$, which are defined on intervals $[0, \infty]$ and $[0,1]$, respectively.

In order to evaluate $I_{0}(t)$, a change of variable $\gamma=t^{\alpha \epsilon} x^{-\frac{\alpha}{2}}$ is employed, and in case of $\hat{T} \neq T$, 
we obtained

$$
I_{0}(t)=\frac{2 t^{\epsilon}}{\alpha} \frac{1}{s_{1}-s_{2}} \int_{0}^{\infty}\left[\frac{s_{1}^{2} \gamma^{-\frac{2}{\gamma}}}{1+s_{1} \gamma}-\frac{s_{2}^{2} \gamma^{-\frac{2}{\gamma}}}{1+s_{2} \gamma}\right] d \gamma
$$

The integral can be separated into two integrals, which are evaluated by employing changes of variables $\gamma_{1}=s_{1} \gamma$ and $\gamma_{2}=s_{2} \gamma$, and following the properties of Gamma function. Consequently, $I_{0}(t)$ is given by

$$
I_{0}(t)=\frac{2 t^{\epsilon}}{\alpha} \frac{s_{1}^{1+\frac{2}{\alpha}}-s_{2}^{1+\frac{2}{\alpha}}}{s_{1}-s_{2}} \frac{\pi}{\sin \left(\frac{2 \pi}{\alpha}\right)}
$$

The integral $I_{1}(t)$ is approximated by using Gauss - Legendre approximation

$$
I_{1}(t)=\sum_{i=1}^{N_{G}} \frac{c i}{2} \frac{\left(s_{1}+s_{2}\right) t^{\alpha \epsilon} \eta_{i}^{\frac{\alpha}{2}}+s_{1} s_{2} t^{\alpha \epsilon}}{\left(\eta_{i}^{\frac{\alpha}{2}}+s_{1} t^{\alpha \epsilon}\right)\left(\eta_{i}^{\frac{\alpha}{2}}+s_{2} t^{\alpha \epsilon}\right)}
$$

Consequently, using the properties of Gauss - Laguerre Quadrature, $v\left(s_{1}, s_{2}, \lambda\right)$ is approximated by

$$
v\left(s_{1}, s_{1}, \lambda\right) \approx \pi \lambda \sum_{m=1}^{N_{G L}} \frac{w_{m}}{2}\left(I_{0}\left(\zeta_{m}\right)-I_{1}\left(\zeta_{m}\right)\right)
$$

Approximate Average Coverage Probability of the CCU. It is clear from (14) that the average coverage probability expression has a suitable form of Gauss - Laguerre. Thus, it can be approximated by

$$
\frac{\sum_{j=1}^{N_{G L}} w_{j} e^{-\frac{(T+\hat{T})}{S N R} \zeta_{j}^{\alpha(1-\epsilon)}} \mathscr{L}\left(\hat{T} \zeta_{j}^{-\alpha \epsilon}, T \zeta_{j}^{-\alpha \epsilon}\right)}{\sum_{j=1}^{N_{G L}} w_{j} e^{-\frac{T}{S N R} \zeta_{j}^{\alpha(1-\epsilon)}} \mathscr{L}_{I_{\theta}^{(o c)}}\left(T \zeta_{j}^{-\alpha \epsilon}\right)}
$$

The proposition is proved.

\section{Appendix E. (Strict FR) The Average Coverage Probability of CEU}

The coverage probability of a CCU under the Strict FR network is obtained based on approach in 10. given that the density of interfering users is $\lambda / \Delta$. Hence,

$$
\mathcal{P}_{c}^{(e)}(T, \epsilon)=\frac{\int_{0}^{\infty} 2 \pi \lambda r e^{-\pi \lambda r^{2}} \mathbb{E}\left[e^{-\frac{\hat{T}\left(\sigma^{2}+I_{\theta}^{(e)}\right)}{P^{(e)} r^{-\alpha}}}\left(1-e^{-\frac{T\left(\sigma^{2}+I_{\theta}^{(o c)}\right)}{P^{(c)} r-\alpha}}\right)\right] d r}{1-\int_{0}^{\infty} 2 \pi \lambda r e^{-\pi \lambda r^{2}} e^{-\frac{T \sigma^{2}}{P^{(c)} r^{-\alpha}}} \mathbb{E}\left[-\frac{T I_{\theta}^{(o c)}}{P^{(c)} r^{-\alpha}} d r\right] d r}
$$

Since the user is defined as the CEU, it will be served on a different RB. Hence, the user experiences new interference from new users. Therefore, the distance from the interfering user to 
it's own serving BS changes from $r_{j}$ to $r_{j e}$ and to the serving BS of user $z$ is $d_{z e}$. We denote $s_{1}^{\prime}=\hat{T} r^{\alpha(1-\epsilon)}$ and $s_{2}^{\prime}=\operatorname{Tr}^{\alpha(1-\epsilon)}$, the numerator can be evaluated as below:

$$
\begin{aligned}
& \stackrel{(\mathrm{a})}{=} \prod_{j \in \theta_{e}} \mathbb{E}\left[\frac{v\left(\frac{\hat{T}}{\phi}\right)}{1+s_{2}^{\prime} r_{j}^{\epsilon \alpha} d_{j e}^{-\alpha}}\right]\left(1-\prod_{j \in \theta} \mathbb{E}\left[\frac{e^{-T r^{\alpha(1-\epsilon)}}}{1+s_{1}^{\prime} r_{j}^{\epsilon \alpha} d_{j z}^{-\alpha}}\right]\right) \\
& \stackrel{(\mathrm{b})}{=} v\left(\frac{\hat{T}}{\phi}\right) e^{-\frac{2 \pi \lambda}{\Delta} \int_{r}^{\infty}\left[1-\int_{0}^{\infty} \frac{2 \pi \lambda t}{\Delta} \frac{-\frac{\pi \lambda t^{2}}{\Delta}}{1+s_{2}^{\prime} t^{\epsilon \alpha} d_{j e}^{-\alpha}} d t\right] d_{j e} d\left(d_{j e}\right)} \\
& \left(1-e^{-T r^{\alpha(1-\epsilon)}} e^{-2 \pi \lambda \int_{r}^{\infty}\left[1-\int_{0}^{\infty} \frac{2 \pi \lambda e^{-\pi \lambda t^{2}}}{1+s_{1}^{\prime} 1^{\epsilon \alpha} d_{j z}^{-\alpha}} d t d_{j z} d\left(d_{j z}\right)\right.}\right) \\
& =v\left(\frac{\hat{T}}{\phi}\right) \mathscr{L}_{I_{\theta}^{(o c)}}\left(s_{2}, \frac{\lambda}{\Delta}\right)\left(1-e^{-T r^{\alpha(1-\epsilon)}} \mathscr{L}_{I_{\theta}^{(o c)}}\left(s_{1}, \lambda\right)\right)
\end{aligned}
$$

in which $(a)$ is obtained by assuming that the fading channel has a Rayleigh distribution and by

400 denoting $v\left(\frac{\hat{T}}{\phi}\right)=e^{-\frac{\hat{T}}{\hat{S N R}} r^{\alpha(1-\epsilon)}}, s_{1}=\operatorname{Tr} r^{\alpha \epsilon}$ and $s_{2}=\hat{T} r^{\alpha \epsilon} ;(b)$ is obtained by taking expectation with respects to $r_{j e}$ and $r_{j}$ and follows by the properties of PGF. $\mathscr{L}_{I_{\theta}^{(o c)}}(s)$ is defined in 77

\section{Appendix F. Soft FR: The average coverage probability of the CCU}

The average coverage probability of the CCU in Soft FR is given by

$$
\begin{aligned}
& \mathcal{P}_{c}^{(c)}(T, \epsilon)=\frac{\mathbb{P}\left(\frac{P^{(c)} g r^{-\alpha}}{\sigma^{2}+I_{\text {Sof }}^{(c)}}>\hat{T}, \frac{P^{(c)} g^{(o)} r^{-\alpha}}{\sigma^{2}+I_{\text {Sof }}^{(o c)}}>T\right)}{\mathbb{P}\left(\frac{P g r^{\alpha(\epsilon-1)}}{\sigma^{2}+I_{\text {Sof }}^{(o c)}}>T\right)} \\
& =\frac{\int_{0}^{\infty} r e^{-\pi \lambda r^{2}} e^{-\frac{(T+\hat{T}) \sigma^{2}}{P^{(c)} r^{-\alpha}}} \mathbb{E}\left[e^{-\frac{\hat{T} I_{S o f}^{(c)}}{P^{(c)} r_{r}^{-\alpha}}-\frac{T I_{S o f}^{(o)}}{P^{(c)} r^{-\alpha}}}\right] d r}{\int_{0}^{\infty} r e^{-\pi \lambda r^{2}}\left(e^{-\frac{T \sigma^{2}}{P^{(c)} r_{r}-\alpha}} \mathbb{E}\left[-\frac{T I_{\text {Sof }}^{(o c)}}{P^{(c)} r^{-\alpha}} d r\right]\right) d r}
\end{aligned}
$$

The expectation of the numerator in $(\mathrm{F} .1)$ is the joint Laplace transform $\mathscr{L}_{\text {Sof }}\left(s_{1}^{\prime}, s_{2}^{\prime}\right)$ of interferences during the establishment phase communication phase in which $s_{1}^{\prime}=\operatorname{Tr}^{\alpha(1-\epsilon)}$ and $s_{2}^{\prime}=\hat{T} r^{\alpha(1-\epsilon)}$. By using the definition of $I_{\text {Sof }}^{(z)}$ in $44, \mathscr{L}_{\text {Sof }}\left(s_{1}^{\prime}, s_{2}^{\prime}\right)=$

$$
=\mathbb{E}\left[e^{-\sum_{j \in \theta_{S o f}^{(c)}}\left(s_{2}^{\prime} r_{j}^{\alpha \epsilon} d_{j z}^{-\alpha} g_{j z}+s_{1}^{\prime} r_{j}^{\alpha \epsilon} d_{j z}^{-\alpha} g_{j z}^{(o)}\right)-\sum_{j \in \theta_{S o f}^{(e)}}\left(\phi s_{2}^{\prime} r_{j}^{\alpha \epsilon} d_{j z}^{-\alpha} g_{j z}+\phi s_{1}^{\prime} r_{j}^{\alpha \epsilon} g_{j z} d_{j z}^{-\alpha} g_{j z}^{(o)}\right)}\right]
$$


Since each BS in $I_{\text {Sof }}^{(c)}$ is distributed independently to any BS in $I_{\text {Sof }}^{(e)}$ and all channels are independent Rayleigh fading channels, $\mathscr{L}_{\text {Sof }}\left(s_{2}^{\prime}, s_{1}^{\prime}\right)=$

$$
\begin{aligned}
& =\prod_{j \in \theta_{\text {Sof }}^{(c)}} \mathbb{E}\left[e^{-\left(s_{2}^{\prime} r_{j}^{\alpha \epsilon} d_{j z}^{-\alpha} g_{j z}+s_{1}^{\prime} r_{j}^{\alpha \epsilon} d_{j z}^{-\alpha} g_{j z}^{(o)}\right)}\right] \prod_{j \in \theta_{\text {Sof }}^{(e)}} \mathbb{E}\left[e^{-\left(\phi s_{2}^{\prime} r_{j}^{\alpha \epsilon} d_{j z}^{-\alpha} g_{j z}+\phi s_{1}^{\prime} r_{j}^{\alpha \epsilon} d_{j z}^{-\alpha} g_{j z}^{(o)}\right)}\right] \\
& =\prod_{j \in \theta_{\text {Sof }}^{(c)}} \mathbb{E}\left[\frac{1}{1+s_{2}^{\prime} r_{j}^{\alpha \epsilon} d_{j z}^{-\alpha}} \frac{1}{1+s_{1}^{\prime} r_{j}^{\alpha \epsilon} d_{j z}^{-\alpha}}\right] \prod_{j \in \theta_{\text {Sof }}^{(e)}} \mathbb{E}\left[\frac{1}{1+\phi s_{2}^{\prime} r_{j}^{\alpha \epsilon} d_{j z}^{-\alpha}} \frac{1}{1+\phi s_{1}^{\prime} r_{j}^{\alpha \epsilon} d_{j z}^{-\alpha}}\right]
\end{aligned}
$$

Given that $r_{j}$ is the distance from user $j$ to it's serving BS, whose PDF follows (1), and using the properties of PGF with respect to variable $d_{j z}$ over $I_{S o f}^{(c)}$ and $I_{S o f}^{(e)}$, the joint Laplace transform $\mathscr{L}_{\text {Sof }}\left(s_{2}^{\prime}, s_{1}^{\prime}\right)$ is given by

$$
\begin{gathered}
=\prod_{j \in \theta_{\text {Sof }}^{(c)}} \mathbb{E}\left[\int_{0}^{\infty} \frac{\frac{2 \pi(\Delta-1) \lambda}{\Delta} t e^{-\frac{\pi(\Delta-1) \lambda}{\Delta} t^{2}}}{\left(1+s_{2}^{\prime} t^{\alpha \epsilon} d_{j z}^{-\alpha}\right)\left(1+s_{1}^{\prime} t^{\alpha \epsilon} d_{j z}^{-\alpha}\right)}\right] \prod_{j \in \theta_{\text {Sof }}^{(e)}} \mathbb{E}\left[\int_{0}^{\infty} \frac{\frac{2 \pi \lambda}{\Delta} t e^{-\frac{\pi \lambda}{\Delta} t^{2}}}{\left(1+\phi s_{2}^{\prime} t^{\alpha \epsilon} d_{j z}^{-\alpha}\right)\left(1+\phi s_{1}^{\prime} t^{\alpha \epsilon} d_{j z}^{-\alpha}\right)}\right] \\
=e^{-\frac{2 \pi(\Delta-1)}{\Delta} \lambda \int_{r}^{\infty}\left[1-\int_{0}^{\infty} \frac{\frac{2 \pi(\Delta-1) \lambda}{\Delta} t e^{-\frac{\pi(\Delta-1) \lambda}{\Delta} t^{2}}}{\left(1+s_{2}^{\prime} t^{\alpha \epsilon} d_{j z}^{-\alpha}\right)\left(1+s_{1}^{\prime} t^{\alpha \epsilon} d_{j z}^{-\alpha}\right)}\right] d_{j z} d\left(d_{j z}\right)} \\
e^{-\frac{2 \pi}{\Delta} \lambda \int_{r}^{\infty}\left[1-\int_{0}^{\infty} \frac{\frac{2 \pi \lambda}{\Delta} t e^{-\frac{\pi \lambda}{\Delta} t^{2}}}{\left(1+\phi s_{2}^{\prime} t^{\alpha \epsilon} d_{j z}^{-\alpha}\right)\left(1+\phi s_{1}^{\prime} t^{\alpha \epsilon} d_{j z}^{-\alpha}\right)}\right] d_{j z} d\left(d_{j z}\right)}
\end{gathered}
$$

By letting $s_{2}=\hat{T} r^{-\alpha \epsilon}$ and $s_{1}=T r^{-\alpha \epsilon}$, and using the change of variable $x=\frac{d_{j z}}{r}$, the joint Laplace transform $\mathscr{L}_{\text {Sof }}\left(s_{2}, s_{2}\right)$

$$
\begin{aligned}
& =e^{-\frac{2 \pi(\Delta-1) \lambda r^{2}}{\Delta} \int_{1}^{\infty}\left[1-\int_{0}^{\infty} \frac{\frac{2 \pi(\Delta-1) \lambda}{\Delta} t e^{-\frac{\pi(\Delta-1) \lambda}{\Delta} t^{2}}}{\left(1+s_{2} t^{\alpha \epsilon} d_{j z}^{-\alpha}\right)\left(1+s_{2} t^{\alpha \epsilon} x^{-\alpha}\right)}\right] d x e^{-\frac{2 \pi \lambda r^{2}}{\Delta} \int_{1}^{\infty}\left[1-\int_{0}^{\infty} \frac{\frac{2 \pi \lambda}{\Delta} t e^{-\frac{\pi \lambda}{\Delta} t^{2}}}{\left(1+\phi s_{2} t^{\alpha \epsilon} d_{j z}^{-\alpha}\right)\left(1+\phi s_{2} t^{\alpha \epsilon} x^{-\alpha}\right)}\right] d x}} \\
& =\mathscr{L}\left(s_{1}, s_{2}, \frac{\Delta-1}{\Delta} \lambda\right) \mathscr{L}\left(\phi s_{1}, \phi s_{2}, \frac{\lambda}{\Delta}\right)
\end{aligned}
$$

By substituting $(\mathrm{F} .2)$ into $\mathrm{F} .1 \mathrm{~T}$ and remind that the denominator is given by Appendix A, the Theorem is proved.

The approximated value of the average coverage probability is obtained by using a change of variable $\zeta=\pi \lambda r^{2}$ and Gauss-Laguerre Quadrature. 


\section{Appendix G. Soft FR: The average coverage probability of the CEU}

The average coverage probability of the CEU in Soft FR is given by

$$
\begin{aligned}
\mathcal{P}_{c}^{(e)}(T, \epsilon)= & \frac{\mathbb{P}\left(\frac{P^{(e) g r^{-\alpha}}}{\sigma^{2}+I_{\text {Sof }}^{(e)}}>\hat{T}, \frac{P^{(c)} g^{(o)} r^{-\alpha}}{\sigma^{2}+I_{\text {Sof }}^{(o)}}<T\right)}{\mathbb{P}\left(\frac{P g r^{\alpha(\epsilon-1)}}{\sigma^{2}+I_{\text {Sof }}^{(o c)}}<T\right)} \\
= & \frac{\int_{0}^{\infty} 2 \pi \lambda r e^{-\pi \lambda r^{2}} \mathbb{E}\left[e ^ { - \frac { \hat { T } ( \sigma ^ { 2 } + I _ { \text { Sof } } ^ { ( e ) } ) } { P ^ { ( e ) } - \alpha } } \left(1-e^{\left.\left.-\frac{T\left(\sigma^{2}+I_{S o f}^{(o c)}\right)}{P^{(c)}-\alpha}\right)\right] d r}\right.\right.}{1-\int_{0}^{\infty} 2 \pi \lambda r e^{-\pi \lambda r^{2}} e^{-\frac{T \sigma^{2}}{P^{(c)} r_{r}-\alpha}} \mathbb{E}\left[-\frac{T I_{\theta}^{(o c)}}{P^{(c)} r^{-\alpha}} d r\right] d r}
\end{aligned}
$$

Since the interfering sources during the communication phase are distributed completely independently to those during the establishment phase, the average coverage probability can be re-written in the following form

$$
\mathcal{P}_{c}^{(e)}(T, \epsilon)=\frac{\int_{0}^{\infty} 2 \pi \lambda r e^{-\pi \lambda r^{2}} \mathbb{E}\left[e^{-\frac{\hat{T}\left(\sigma^{2}+I_{\text {Sof }}^{(e)}\right)}{\phi P^{(c)} r^{-\alpha}}}\right]\left(1-\mathbb{E}\left[e^{-\frac{T\left(\sigma^{2}+I_{S o f}^{(o c)}\right)}{P^{(c)} r^{-\alpha}}}\right]\right) d r}{1-\int_{0}^{\infty} 2 \pi \lambda r e^{-\pi \lambda r^{2}} e^{-\frac{T \sigma^{2}}{\left.P^{(c)}\right)^{-\alpha}}} \mathbb{E}\left[-\frac{T I_{\theta}^{(o c)}}{P^{(c)} r^{-\alpha}} d r\right] d r}
$$

Using the results of Appendix A the Theorem 3.5 is proved.

\section{References}

[1] A. S. Hamza, S. S. Khalifa, H. S. Hamza, K. Elsayed, A Survey on Inter-Cell Interference Coordination Techniques in OFDMA-Based Cellular Networks, IEEE Commun. Surveys \& Tutorials 15 (4) (2013) 1642-1670. doi:10.1109/SURV.2013.013013.00028.

[2] 3GPP TS 36.214 V9.1.0 Release 9, LTE; Evolved Universal Terrestrial Radio Access (EUTRA); Physical layer - Measurements, 2010.

[3] 3GPP TS 36.213 V8.8.0 Release 8, LTE; Evolved Universal Terrestrial Radio Access (EUTRA); Physical layer procedures, 2009.

[4] HUAWEI TECHNOLOGIES CO., LTD, eLTE2.2 DBS3900 LTE FDD Basic Feature Description, 2014.

[5] O. Somekh, S. Shamai, Shannon-theoretic approach to a Gaussian cellular multiple-access channel with fading, IEEE Trans. Inf. Theory 46 (4) (2000) 1401-1425. doi:10.1109/18. 850679. 
[6] H. ElSawy, E. Hossain, M. Haenggi, Stochastic Geometry for Modeling, Analysis, and Design of Multi-Tier and Cognitive Cellular Wireless Networks: A Survey, IEEE Commun. Surveys Tutorials 15 (3) (2013) 996-1019. doi:10.1109/SURV.2013.052213.00000.

[7] T. D. Novlan, R. K. Ganti, A. Ghosh, J. G. Andrews, Analytical Evaluation of Fractional Frequency Reuse for OFDMA Cellular Networks, IEEE Trans. Wireless Commun. 10 (2011) $4294-4305$.

[8] H. Zhuang, T. Ohtsuki, A Model Based on Poisson Point Process for Analyzing MIMO Heterogeneous Networks Utilizing Fractional Frequency Reuse, IEEE Trans. Wireless Commun. 13 (12) (2014) 6839-6850. doi:10.1109/TWC.2014.2364219.

[9] Y. Lin, W. Yu, Optimizing user association and frequency reuse for heterogeneous network under stochastic model, in: 2013 IEEE Global Commun. Conf.(GLOBECOM), 2013, pp. 2045-2050. doi:10.1109/GLOCOM.2013.6831376.

[10] T. D. Novlan, J. G. Andrews, Analytical Evaluation of Uplink Fractional Frequency Reuse, IEEE Trans. Commun. 61 (5) (2013) 2098-2108. doi:10.1109/TCOMM. 2013.031213.120260.

[11] F. J. Martn-Vega, F. J. Lpez-Martnez, G. Gmez, M. C. Aguayo-Torres, Multi-user coverage probability of uplink cellular systems: A stochastic geometry approach, in: 2014 IEEE Global Communi. Conf. (GLOBECOM), 2014, pp. 3989-3994.

[12] W. Bao, B. Liang, Stochastic Analysis of Uplink Interference in Two-Tier Femtocell Networks: Open Versus Closed Access, IEEE Trans. Wireless Commun. 14 (11) (2015) 6200-6215. doi: 10.1109/TWC.2015.2450216.

[13] 3GPP Release 10 V0.2.1, LTE-Advanced (3GPP Release 10 and beyond), 2014.

[14] S. C. Lam, R. Subramanian, K. Sandrasegaran, P. Ghosal, S. Barua, Performance of wellknown frequency reuse algorithms in LTE downlink 3GPP LTE systems, in: Proc. IEEE Int. Conf. Signal Process. and Communication Syst.(ICSPCS), 2015, pp. 1-5. doi:10.1109/ ICSPCS. 2015.7391766.

[15] H. Zhuang, T. Ohtsuki, A model based on Poisson point process for downlink K tiers fractional frequency reuse heterogeneous networks, Physical Communication Volume 13, Part B (Special Issue on Heterogeneous and Small Cell Networks) (2014) 312.

${ }_{450}[16]$ S. C. Lam, K. Sandrasegaran, T. N. Quoc, Performance of soft frequency reuse in random cellular networks in Rayleigh-Lognormal fading channels, in: 2016 22nd Asia-Pacific Conf. Commun. (APCC), 2016, pp. 481-487. doi:10.1109/APCC.2016.7581454. 
[17] S. C. Lam, K. Sandrasegaran, P. Ghosal, Performance analysis of frequency reuse for ppp networks in composite rayleigh-lognormal fading channel, Wireless Personal Communications doi: $10.1007 / \mathrm{s} 11277-017-4215-2$.

[18] H. B. Chang, I. Rubin, Optimal downlink and uplink fractional frequency reuse in cellular wireless networks, IEEE Trans. on Veh. Tech. 65 (4) (2016) 2295-2308.

[19] Zhixin Liu and Shiyi Li and Lili Hao and Hongjiu Yang and Xinping Guan and Xinbin $\mathrm{Li}$, Power control based on the stackelberg game in two-tier femtocell networks, Physical Communication 23 (2017) 1 - 11. doi:http://doi.org/10.1016/j.phycom.2017.01.005.

[20] F. C. Ribeiro, R. Dinis, F. Cercas, A. Silva, Multiuser detection for the uplink of clustered 5G systems with universal frequency reuse, Physical Communication 23 (2017) 29 - 36. doi: http://doi.org/10.1016/j.phycom.2017.01.006.

[21] T. D. Novlan, H. S. Dhillon, J. G. Andrews, Analytical modeling of uplink cellular networks, IEEE Trans. Wireless Commun. 12 (6) (2013) 2669-2679. doi:10.1109/TWC.2013.050613. 120325 .

[22] Technical specification group radio access network; evolved universal terrestrial radio access (e-utra);physical layer procedures (2009).

[23] Kesan Online Calculator[link], URL http://keisan.casio.com/

[24] 3GPP TR 36.828 V11.0, E-UTRA Further enhancements to LTE Time Division Duplex (TDD) for Downlink-Uplink (DL-UL) interference management and traffic adaptation, 2012.

[25] J. G. Andrews, F. Baccelli, R. K. Ganti, A new tractable model for cellular coverage, in: 2010 48th Annu. Allerton Conference on Communication, Control, and Computing (Allerton), pp. 1204-1211. doi:10.1109/ALLERTON.2010.5707051.

[26] 3GPP TS 36.211 V14.1.0, E-UTRA Physical Channels and Modulation, 2016.

[27] M. A. Stegun, I. A., Handbook of Mathematical Functions with Formulas, Graphs, and Mathematical Tables, Dover Publications, 1972. 